\title{
Development, Item Analysis and Standardization of Teachers Cognitive Ability Test
}

\author{
H.K. Nanda ${ }^{1}$, Shruti Marwaha ${ }^{1 *}$, Pawandeep Chawla ${ }^{1}$
}

\section{ABSTRACT}

The present research study was conducted for the development, item analysis and standardization of Teachers Cognitive Ability Test. Random sampling procedure was followed to select 383 subjects from different cities. Subjects consisted of male as well as female in-service teachers. The objective of the research was achieved in the form of a reliable and valid test intended to provide an insight into those scientific methodologies that can help us measure and reorder human intelligence to enhance cognitive factors among teachers by filling the gaps, to produce successful and efficient teachers. The validity was estimated through linear regression method. The reliability was calculated via Test-Retest Method. The main objective of the study was to develop test items through Bloom's taxonomy, Leslie Wilson theory, determining the Difficulty, Discrimination Index \& Reliability. The test would help in the identification, measurement and analysis of core cognitive ability factors that determine success in teaching.

Keywords: Development and Standardization, Cognitive Ability, Bloom's taxonomy, Leslie Wilson theory, Reliability, Validity, Discrimination Index

Teachers need effective ways to focus on their core task of improving teaching and learning. Educational improvement must be practical and collaborative. Even if schools and students differ, when teachers work together, student learning improves. The traditional professional development practices were largely based on transferring knowledge from an expert to an audience. But, these days overall Career advancement for teachers is one of the central concerns of reformers. Tracing and retaining the best qualified teachers will require the development of systems that properly reward and empower classroom teachers. Mertens and Yarger examine the issues associated with career ladder opportunities and question a selected set of assumptions that undergird professional enhancement schemes. The authors conclude with a discussion of how teacher empowerment and involvement are essential ingredients to strengthening teaching as a profession.Good teachers form the foundation of good schools, and improving teachers' skills and knowledge is one of the most important investments of time and money that local, state, and national leaders make in education. Researches reveal that students of teachers who participated in this kind of curriculum-focused professional development did well on assessments.

\footnotetext{
${ }^{1}$ Department of Research and Development, MaxproIntellithon Ltd.

*Corresponding Author

(C) 2015 I H Nanda, S Marwaha, P Chawla; licensee IJIP. This is an Open Access Research distributed under the terms of the Creative Commons Attribution License (http://creativecommons.org/licenses/by/2.0), which permits unrestricted use, distribution, and reproduction in any Medium, provided the original work is properly cited.
} 
Unfortunately, most teachers received less effective forms of training. Studies suggest that the more time teachers spend on professional development, the more significantly they change their practices and that participating in professional learning communities optimizes the time spent on professional development. A Piagetian approach to cognitive development assumes that cognitive development is independent from language development. Information enters the mind to stimulate cognitive development through perception of sound, visual information, speech, and touch. Cognitive organization helps to store and remember concepts. Learners are like scientists, trying to explore and figure out how the world works based on what they see, do, and hear. Teaching is an art, which must be learnt before launching ourselves into the dynamism of School life. It is the combination of structured planning and choices. The outcome of a successful career in teaching should include personal fulfillment, work/life balance, goal achievement and financial assurance. Most of the time, we opt for teaching career based on our professional qualification \& academic performances, only to find out in later years that we were meant to do something else. This can drastically enhance the learning process of individuals. Thus, evaluation in its current form generally contributes little either to teacher learning or to accurate, timely information for personnel decisions. In the context of the current interest in measuring teacher effectiveness, it is important to distinguish between teacher quality and teaching quality. Teacher quality is a bundle of personal traits, skills, and understanding that an individual brings to teaching, including dispositions to behave in certain ways. Researches on teacher effectiveness, based on teacher ratings and student achievement gains, have found that qualitative teaching is essential for composed learning leading to serenity in personal and professional life.

\section{REVIEW OF LITERATURE}

Amelioration in the goals for learning, coupled with shifts in curriculum emphasis and a deeper understanding of teacher learning and student thinking, have led to new findings about the impact of teacher professional development and how best to sharpen teachers' skills and knowledge. To be effective, professional development must provide teachers with a way to directly apply what they learn to their teaching. Research shows that professional development leads to better instruction and improved student learning when it connects to the curriculum materials that teachers use, the district and state academic standards that guide their work, and the assessment and accountability measures that evaluate their success. Professional development should improve teachers' knowledge of the subject matter that they are teaching, and it should enhance their understanding of student thinking in that subject matter. Teacher quality is the most important school factor for improving student cognitive ability. Researchers have found that variation in student achievement is explained more by variation in teacher quality than variation in any other school characteristics (Rivkin, Hanushek, \&Kain 2005; Rockoff 2004). Research indicates that high-quality teachers, as measured by their ability to raise student math and reading test scores, improve longer-run outcomes such as their students' educational attainment and employment income (Chetty, Friedman, Rockoff 2014). Nonetheless, scholars are generally unable to identify high quality teachers based upon observable 
characteristics absent measures of student achievement. For instance, years of teaching experience is generally uncorrelated with teacher quality after the first three to five years of teaching (Boyd et al. 2007; Buddin \& Zamarro 2009; Hanushek \& Rivkin 2006; Clotfelter, Ladd, \&Vigdor 2006; Goldhaber 2007; Kane, Rockoff, \&Staiger 2008). Teacher licensure (e.g., whether a teacher has a Master's degree or completed a traditional certification program) is likewise not strongly correlated with a teacher's ability to raise student scores on achievement tests (Hanushek \& Rivkin 2006; Podgursky 2005; Hanushek 1997). Although there is some evidence that having more content knowledge, as measured by the number of courses taken in that content area, is associated with higher teacher quality, this relationship largely holds for secondary school teachers, particularly in math or science (Clotfelter, Ladd, \& Vigdor 2006; 6 Goldhaber\& Brewer 2000; Monk \& King 1994). There is also a lack of evidence that pedagogical knowledge for a specific content area is linked with student achievement (Hill, Rowan, \& Ball 2005). Although some research has demonstrated that achievement is higher for students with teachers that have higher cognitive ability, as measured by their performance on the Praxis or other standardized licensure tests (Goldhaber 2007; Clotfelter, Ladd, \&Vigdor 2006), other work finds no relationship between teacher cognitive ability and student achievement (Buddin\&Zamarro 2009). Finally, Duckworth, Quinn, and Selgiman (2009) provide suggestive evidence that some teacher non-cognitive abilities (e.g., grit and life satisfaction) are positively correlated with student gains in cognitive ability. However, their analysis is based upon a convenience sample of an atypical group of teachers - first- and second-year Teach for America teachers. In summary, research suggests that teacher quality matters for student wellbeing, but it is difficult to predict teacher quality solely based on teacher inputs and observable characteristics. This has led to some proposals to relax the selection of teachers based upon inputs (e.g., credentials) and to evaluating teachers based upon their outputs or actual performance (e.g., student achievement) (Podgursky 2005; Goldhaber 2008; Kane, Rockoff, \&Staiger 2008; Hanushek 2011). Presumably, certain pedagogical practices could shape student perception and mindset, ultimately affecting student behavior (Dweck 2006). Certain pedagogical practices may also lead to unique classroom environments or climates, which can, for example, alter student motivations to work towards a particular goal and induce students to 10 exert the necessary self-regulation towards that end (Ames 1992). Organizational features such as school or class size may also be important for cognitive skill development (Osterman 2000). Smaller class sizes may enable teachers to better motivate and engage their students. It may be easier for teachers to manage smaller classes, enabling them to more firmly establish salient values and norms that affect the cognitive skill development of their students. Indeed, data from National Education Longitudinal Study of 1988 indicate that middle-school students in smaller classes tend to be more motivated and engaged in learning (Dee \& West 2011). Overall, research on cognitive skills is relatively nascent and has merely established the importance of cognitive skills for student outcomes. Sociologists have long observed that all schools have values and social norms that reinforce those values (Bryk\& Driscoll 1988; Coleman \& Hoffer 1987). In turn, these values and norms delineate behaviors and attitudes that are appropriate or inappropriate (Bryk et al. 1993). Students may develop particular dispositions and personality 
traits as they are socialized according to these values and norms. Psychologists have long proposed that learning is social (Bandura 1977). Some have more specifically argued that individuals learn group norms by observing the behaviors of other group members, called social referents, in specific situations. A social referent helps individuals discern what types of behaviors are acceptable or unacceptable by allowing them to observe what behaviors are rewarded or sanctioned within the group (Sherif\&Sherif 1964). Social referents in schools may consist of a student's peer group or teachers (Paluck\& Shepherd 2012). Teachers 11 are particularly well-situated to act as role models, instilling a set of traits derived from a certain value system into their students. It is in this way that individual teachers can possibly influence the cognitive as well as non-cognitive skills of their students (Berkowitz \& Bier 2004; Lumpkin 2008). The fostering of these skills is hypothesized to explain the higher levels of educational attainment among Catholic school students, even those from disadvantaged backgrounds (Altonji et al; 2005; Coleman \& Hoffer 1987; Evans \& Schwab 1995; Grogger\& Neal 2000; Neal 1997; Sander \&Krautman 1995; Sander 2001). The results, especially those concerning non-test score outcomes, are consistent with the research on cognitive skills and their role in health, crime, and attainment outcomes (Almlund, et al. 2011; Heckman et al. 2006; Heckman \& Rubinstein 2001).

\section{METHODOLOGY}

The test consists of 80 Objective Test questions, and time bound for 20 minutes that is to be taken by the Teacher. Random sampling procedure was followed to select 383 subjects from different cities. Subjects consisted of males as well as females as illustrated in Fig.1

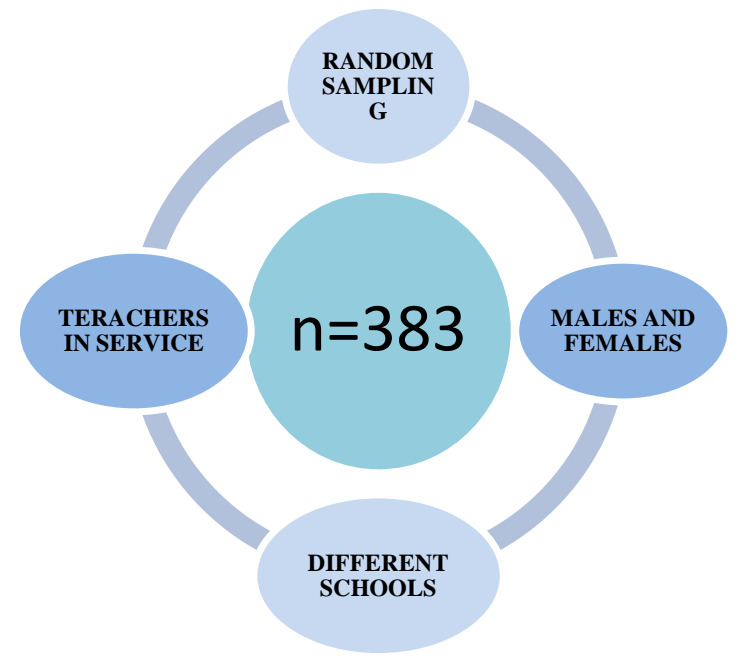

Fig.2Sampling procedure

Test results would include current cognitive capacity measure, dynamic IQ, Focus Factor, Decision Making Ability, CQ, Skill Estimation Level, Natural Learning Style, Cognitive Gaps Measurement, Gifted Ability, and Suitable Career Path.

\section{PROCEDURE AND ADMINISTRATION}

The test consists of 80 items. Rapport building was followed by giving the in-length instructions to the subjects. The specific time in which teachers were required to complete the 
test was allotted and a stop watch was used for the purpose. The required material, i.e. Photocopies of test items, paper, pencil etc. was provided. Test administration procedure was similar for all the participants. Test were marked using standard procedure in which score +1 was given for each item passed. Thus total 80 marks were assigned for each test. Standardization implies uniformity of procedures in administrating and scoring the test. If the scores obtained by different persons are to be comparable, testing conditions must obviously be the same for all. The formulation of directions is a major part of the standardization of a new test. Such standardization extends to the exact materials employed, time limits, oral instructions, preliminary demonstrations, way of handling queries from test takers and every other detail of the testing situation. Another important step in the standardization of a test is the establishment of norms. As its name implies, a norm is the normal or average performance. In the process of standardization a test, it is administrated to a large, representative sample of the type of persons for whom it is designed. This group known as the standardization sample serves to establish the norms, corresponding to the performance of typical or average persons. To estimate and ensure validity, linear regression method was followed. The reliability was estimated by calculating the reliability correlation coefficient. The test-retest method was used to estimate the reliability of the test.

\section{DATA ANALYSIS}

After collecting the data, it was arranged in tabular form and following mention statistical techniques used for items

Table 1: Item Analysis Methods

\begin{tabular}{|l|l|}
\hline \multirow{3}{*}{1} & Item analysis through Leslie Wilson \\
\cline { 2 - 3 } & LOTS - Lower Order Thinking Skill \\
\cline { 2 - 3 } & MOTS - Middle Order Thinking Skill \\
\cline { 2 - 3 } & HOTS - High Order Thinking Skill \\
\hline 2 & Item analysis through Bloom's Taxonomy \\
\hline 3 & Item difficulty level \\
\hline 4 & Index of discrimination \\
\hline 5 & Split half method \\
\hline 6 & Analysis of Validity by Linear Regression \\
\hline
\end{tabular}

To find the item reflect the knowledge, comprehension, application, analysis, synthesis \& evaluation.

To find the difficulty level and discrimination index the test score divided into three groups highest $27 \%$, middle $46 \%$, lowest $27 \%$ percentage.

The difficulty level was calculated with the help of formula

$$
P=\frac{V_{p}}{V}
$$


Where: NP indicates the number of test of test takers in the total group who pass the items, and $\mathrm{N}$ indicates the total number of test takers in the group.

The formula of the item - discrimination Index is:

$$
D=\frac{U_{p}-L_{p}}{U}
$$

Where: Up and LP indicates the numbers of test takers in the upper and lower groups who pass the items, and $\mathrm{U}$ is the total numbers of the test takers in upper group.

The discrimination index was determined by the difference between the percentages of the students doing the item right in the high achieves and low achieves group discrimination index.

\section{Presentation and Analysis of Data}

Summary presentation in tabular form: - ITEM ANALYSIS.

Table 2: Summary of Leslie Owen Wilson

\begin{tabular}{|c|c|c|c|c|c|}
\hline & \multicolumn{5}{|c|}{ Type of Questions Age 7-16 Years Acc To Leslie Owen Wilson } \\
\hline S.no & Factual & Convergent & Divergent & Evaluative & Combination \\
\hline 1 & 1 & & & & \\
\hline 2 & & 1 & & & \\
\hline 3 & & & & 1 & \\
\hline 4 & 1 & & & & \\
\hline 5 & & & & 1 & \\
\hline 6 & & 1 & & & \\
\hline 7 & 1 & & & & \\
\hline 8 & & & & 1 & \\
\hline 9 & & & & $\overline{1}$ & \\
\hline 10 & 1 & & & & \\
\hline 11 & & 1 & & & \\
\hline 12 & & 1 & & & \\
\hline 13 & & & & 1 & \\
\hline 14 & & 1 & & & \\
\hline 15 & 1 & & & & \\
\hline 16 & & 1 & & & \\
\hline 17 & & 1 & & & \\
\hline 18 & & & & 1 & \\
\hline 19 & & 1 & & & \\
\hline 20 & 1 & & & & \\
\hline 21 & & & & 1 & \\
\hline 22 & & & 1 & & \\
\hline 23 & 1 & & & & \\
\hline 24 & & $\overline{1}$ & & & \\
\hline
\end{tabular}


Development, Item Analysis and Standardization of Teachers Cognitive Ability Test

\begin{tabular}{|c|c|c|c|c|c|c|}
\hline 25 & & & 1 & & & \\
\hline 26 & & & 1 & & & \\
\hline 27 & & & & & 1 & \\
\hline 28 & & & & & & 1 \\
\hline 29 & & & 1 & & & \\
\hline 30 & & & & & 1 & \\
\hline 31 & & & & 1 & & \\
\hline 32 & & 1 & & & & \\
\hline 33 & & & 1 & & & \\
\hline 34 & & & & & 1 & \\
\hline 35 & & & & & & 1 \\
\hline 36 & & & 1 & & & \\
\hline 37 & & 1 & & & & \\
\hline 38 & & & 1 & & & \\
\hline 39 & 1 & & & & & \\
\hline 40 & 1 & & & & & \\
\hline 41 & & & 1 & & & \\
\hline 42 & 1 & & & & & \\
\hline 43 & & & & 1 & & \\
\hline 44 & 1 & & & & & \\
\hline 45 & & & 1 & & & \\
\hline 46 & & & & & 1 & \\
\hline 47 & & & & & 1 & \\
\hline 48 & & & & & 1 & \\
\hline 49 & & & & & $\begin{array}{ll}1 \\
\end{array}$ & \\
\hline 50 & & & & & $\begin{array}{ll}1 \\
\end{array}$ & \\
\hline 51 & & & & & 1 & \\
\hline 52 & & & & & & 1 \\
\hline 53 & & & 1 & & & \\
\hline 54 & & & 1 & & & \\
\hline 55 & & & & & & 1 \\
\hline 56 & & 1 & & & & \\
\hline 57 & 1 & & & & & \\
\hline 58 & 1 & & & & & \\
\hline 59 & 1 & & & & & \\
\hline 60 & & & & & 1 & \\
\hline 61 & & & & & 1 & \\
\hline 62 & 1 & & & & & \\
\hline 63 & & & & & & 1 \\
\hline 64 & & & & & & 1 \\
\hline 65 & & & 1 & & & \\
\hline 66 & $\overline{1}$ & & & & & \\
\hline 67 & 1 & & & & & \\
\hline 68 & & & & & 1 & \\
\hline
\end{tabular}

(C) The International Journal of Indian Psychology | 171 
Development, Item Analysis and Standardization of Teachers Cognitive Ability Test

\begin{tabular}{|c|c|c|c|l|l|}
69 & & & 1 & & \\
\hline 70 & 1 & & & & \\
\hline 71 & & 1 & & & \\
\hline 72 & & 1 & & & \\
\hline 73 & & 1 & & & \\
\hline 74 & & 1 & & & \\
\hline 75 & 1 & & & & \\
\hline 76 & & & 1 & & \\
\hline 77 & & & 1 & & \\
\hline 78 & & & 1 & & \\
\hline 79 & & & 1 & & \\
\hline 80 & 1 & & & & \\
\hline Total & 20 & 28 & 7 & 19 & \\
\hline
\end{tabular}

Table 3: Summary of Bloom's Taxonomy

\begin{tabular}{|c|c|c|c|c|c|c|c|c|c|c|c|}
\hline & Item & Item & Item & Item & Item & Item & Item & Item & Item & Item & TOTAL \\
\hline \begin{tabular}{|l|} 
Level of \\
Learning \\
Outcomes \\
\end{tabular} & 1 & 2 & 3 & 4 & 5 & 6 & 7 & 8 & 9 & 10 & \\
\hline \multicolumn{12}{|l|}{ Knowledge } \\
\hline Recall & & & 1 & & 1 & & 1 & & & 1 & 4 \\
\hline Identify & 1 & 1 & & 1 & & 1 & 1 & & 1 & & 5 \\
\hline \multicolumn{12}{|c|}{ Comprehension } \\
\hline Interpret & & 1 & 1 & 1 & 1 & 1 & & 1 & 1 & & 6 \\
\hline Classify & & & & & & & & & & 1 & 1 \\
\hline \begin{tabular}{|l} 
Comparing \\
\end{tabular} & 1 & & & 1 & 1 & & 1 & & 1 & & 4 \\
\hline \multicolumn{12}{|l|}{ Application } \\
\hline Solve & & & 1 & & 1 & & & & 1 & & 2 \\
\hline Relate & 1 & 1 & 1 & 1 & & 1 & 1 & 1 & & 1 & 8 \\
\hline \multicolumn{12}{|l|}{ Analysis } \\
\hline Analyse & 1 & 1 & & 1 & & 1 & 1 & 1 & 1 & 1 & 7 \\
\hline Discriminate & & & & & 1 & & & & & & 1 \\
\hline \multicolumn{12}{|l|}{ Synthesis } \\
\hline Devise & & 1 & 1 & & 1 & 1 & & 1 & 1 & 1 & 6 \\
\hline \multicolumn{12}{|l|}{ Evaluation } \\
\hline Justify & 1 & 1 & 1 & 1 & 1 & 1 & 1 & 1 & 1 & 1 & 9 \\
\hline TOTAL & 5 & 6 & 7 & 6 & 7 & 6 & 6 & 5 & 7 & 6 & \\
\hline
\end{tabular}


Development, Item Analysis and Standardization of Teachers Cognitive Ability Test

\begin{tabular}{|l|c|c|c|c|c|c|c|c|c|c|c|}
\hline & Item & Item & Item & Item & Item & Item & Item Item & Item & Item & TOTAL \\
\hline $\begin{array}{l}\text { Level of } \\
\text { Learning } \\
\text { Outcomes }\end{array}$ & 11 & 12 & 13 & 14 & 15 & 16 & 17 & 18 & 19 & 20 & \\
\hline Knowledge & & & & & & & & & & & \\
\hline Recall & 1 & & & 1 & 1 & & & 1 & & 1 & 5 \\
\hline Identify & & 1 & 1 & & 1 & 1 & 1 & & 1 & & 6 \\
\hline \multicolumn{1}{c}{ Comprehension } & & & & & & & & & & \\
\hline Interpret & 1 & 1 & 1 & & & 1 & 1 & 1 & 1 & & 7 \\
\hline Classify & & & & 1 & & & & & & & 1 \\
\hline Comparing & & 1 & & & 1 & & 1 & 1 & 1 & 1 & 6 \\
\hline Application & & & & & & & & & & & \\
\hline Solve & 1 & & & & & & 1 & 1 & & 1 & 4 \\
\hline Relate & & 1 & 1 & 1 & 1 & 1 & & & 1 & & 6 \\
\hline Analysis & & & & & & & & & & & \\
\hline Analyse & 1 & & 1 & & & 1 & 1 & & & 1 & 5 \\
\hline Discriminate & & 1 & & 1 & 1 & & & 1 & 1 & & 5 \\
\hline Synthesis & & & & & & & & & & & \\
\hline Devise & 1 & 1 & 1 & 1 & & 1 & 1 & 1 & 1 & 1 & 9 \\
\hline Evaluation & & & & & & & & & & & \\
\hline Justify & 1 & 1 & 1 & 1 & 1 & 1 & 1 & 1 & 1 & 1 & 10 \\
\hline & & & & & & & & & & & \\
\hline TOTAL & $\mathbf{6}$ & $\mathbf{7}$ & $\mathbf{6}$ & $\mathbf{6}$ & $\mathbf{6}$ & $\mathbf{6}$ & $\mathbf{7}$ & $\mathbf{7}$ & $\mathbf{7}$ & $\mathbf{6}$ & \\
\hline
\end{tabular}

\begin{tabular}{|l|c|c|c|c|c|c|c|c|c|c|c|}
\hline & Item & Item & Item & Item & Item & Item & Item & Item & Item & Item & TOTAL \\
\hline $\begin{array}{l}\text { Level of } \\
\text { Ourning } \\
\text { Outcomes }\end{array}$ & 21 & 22 & 23 & 24 & 25 & 26 & 27 & 28 & 29 & 30 & \\
\hline Knowledge & & & & & & & & & & & \\
\hline Recall & & & 1 & & 1 & 1 & & & & & 3 \\
\hline Identify & 1 & 1 & & 1 & 1 & 1 & 1 & 1 & 1 & & 8 \\
\hline \multicolumn{1}{|c|}{ Comprehension } & & & & & & & & & & \\
\hline Interpret & 1 & 1 & & 1 & & & 1 & 1 & 1 & 1 & 7 \\
\hline Classify & & & & & 1 & 1 & & & & & 2 \\
\hline Comparing & & 1 & 1 & & 1 & 1 & & & & & 4 \\
\hline Application & & & & & & & & & & & \\
\hline
\end{tabular}


Development, Item Analysis and Standardization of Teachers Cognitive Ability Test

\begin{tabular}{|l|c|c|c|c|c|c|c|c|c|c|c|}
\hline Solve & & 1 & 1 & & & & & & & & 2 \\
\hline Relate & 1 & & & 1 & & & & & 1 & 1 & 4 \\
\hline Analysis & & & & & & & & & & & \\
\hline Analyse & 1 & 1 & 1 & 1 & & & 1 & 1 & 1 & 1 & 8 \\
\hline Discriminate & & & & & 1 & 1 & 1 & 1 & 1 & & 5 \\
\hline Synthesis & & & & & & & & & & & \\
\hline Devise & 1 & 1 & 1 & 1 & 1 & 1 & 1 & 1 & 1 & 1 & 10 \\
\hline Evaluation & & & & & & & & & & & \\
\hline Justify & 1 & 1 & 1 & 1 & 1 & 1 & 1 & 1 & 1 & 1 & 10 \\
\hline & & & & & & & & & & & \\
\hline TOTAL & $\mathbf{6}$ & $\mathbf{7}$ & $\mathbf{6}$ & $\mathbf{6}$ & $\mathbf{7}$ & $\mathbf{7}$ & $\mathbf{6}$ & $\mathbf{6}$ & $\mathbf{7}$ & $\mathbf{5}$ & \\
\hline
\end{tabular}

\begin{tabular}{|c|c|c|c|c|c|c|c|c|c|c|c|}
\hline & Item & Item & Item & Item & Item & Item & Item & Item & Item & Item & TOTAL \\
\hline $\begin{array}{l}\text { Level of } \\
\text { Learning } \\
\text { Outcomes }\end{array}$ & 31 & 32 & 33 & 34 & 35 & 36 & 37 & 38 & 39 & 40 & \\
\hline \multicolumn{12}{|l|}{ Knowledge } \\
\hline Recall & & & & & & 1 & 1 & & & & 2 \\
\hline Identify & 1 & 1 & 1 & 1 & 1 & 1 & 1 & 1 & 1 & 1 & 10 \\
\hline \multicolumn{12}{|c|}{ Comprehension } \\
\hline Interpret & 1 & & 1 & 1 & 1 & & & 1 & 1 & & 6 \\
\hline Classify & 1 & & & & 1 & 1 & 1 & & & & 4 \\
\hline Comparing & 1 & 1 & 1 & & 1 & 1 & 1 & & 1 & 1 & 8 \\
\hline \multicolumn{12}{|l|}{ Application } \\
\hline \begin{tabular}{|l|} 
Solve \\
\end{tabular} & & & 1 & & & & & & & & 1 \\
\hline Relate & 1 & 1 & & & 1 & & & 1 & 1 & & 5 \\
\hline \multicolumn{12}{|l|}{ Analysis } \\
\hline Analyse & 1 & & 1 & 1 & 1 & & & 1 & & 1 & 6 \\
\hline Discriminate & & & & 1 & & 1 & 1 & & 1 & 1 & 5 \\
\hline \multicolumn{12}{|l|}{ Synthesis } \\
\hline Devise & 1 & 1 & 1 & 1 & 1 & 1 & 1 & 1 & 1 & 1 & 10 \\
\hline \multicolumn{12}{|l|}{ Evaluation } \\
\hline Justify & 1 & 1 & 1 & 1 & 1 & 1 & 1 & 1 & 1 & 1 & 10 \\
\hline TOTAL & 8 & 5 & 7 & 6 & 8 & 7 & 7 & 6 & 7 & 6 & \\
\hline
\end{tabular}


Development, Item Analysis and Standardization of Teachers Cognitive Ability Test

\begin{tabular}{|l|c|c|c|c|c|c|c|c|c|c|c|}
\hline & Item & Item & Item & Item & Item & Item & Item & Item & Item & Item & TOTAL \\
\hline $\begin{array}{l}\text { Level of } \\
\text { Learning } \\
\text { Outcomes }\end{array}$ & 41 & 42 & 43 & 44 & 45 & 46 & 47 & 48 & 49 & 50 & \\
\hline Knowledge & & & & & & & & & & & \\
\hline Recall & & & & 1 & 1 & & & 1 & & 1 & 4 \\
\hline Identify & 1 & 1 & 1 & & 1 & 1 & 1 & 1 & 1 & & 8 \\
\hline \multicolumn{1}{c|}{ Comprehension } & & & & & & & & & & \\
\hline Interpret & 1 & & 1 & & & 1 & & & 1 & 1 & 5 \\
\hline Classify & & & & 1 & 1 & & & & & & 2 \\
\hline Comparing & & 1 & 1 & & 1 & & 1 & 1 & & 1 & 6 \\
\hline Application & & & & & & & & & & & \\
\hline Solve & & & 1 & & & & & 1 & & 1 & 3 \\
\hline Relate & 1 & 1 & & 1 & & 1 & 1 & & 1 & & 6 \\
\hline Analysis & & & & & & & & & & & \\
\hline Analyse & 1 & 1 & 1 & 1 & & 1 & & & 1 & & 6 \\
\hline Discriminate & & & & & 1 & & & 1 & & 1 & 3 \\
\hline Synthesis & & & & & & & & & & & \\
\hline Devise & 1 & & 1 & & 1 & 1 & 1 & 1 & 1 & 1 & 8 \\
\hline Evaluation & & & & & & & & & & & \\
\hline Justify & 1 & 1 & 1 & 1 & 1 & 1 & 1 & 1 & 1 & 1 & 10 \\
\hline & & & & & & & & & & & \\
\hline TOTAL & $\mathbf{6}$ & $\mathbf{5}$ & $\mathbf{7}$ & $\mathbf{5}$ & $\mathbf{7}$ & $\mathbf{6}$ & $\mathbf{5}$ & $\mathbf{7}$ & $\mathbf{6}$ & $\mathbf{7}$ & \\
\hline
\end{tabular}

\begin{tabular}{|l|c|c|c|c|c|c|c|c|c|c|c|}
\hline & Item & Item & Item & Item & Item & Item & Item & Item & Item & Item & TOTAL \\
\hline $\begin{array}{l}\text { Level of } \\
\text { Ouarning } \\
\text { Outcomes }\end{array}$ & 51 & 52 & 53 & 54 & 55 & 56 & 57 & 58 & 59 & 60 & \\
\hline Knowledge & & & & & & & & & & & \\
\hline Recall & & & 1 & 1 & & 1 & 1 & & & 1 & 5 \\
\hline Identify & & 1 & & & 1 & 1 & 1 & 1 & 1 & 1 & 7 \\
\hline \multicolumn{1}{|c|}{ Comprehension } & & & & & & & & & & \\
\hline Interpret & 1 & 1 & 1 & 1 & 1 & & & & 1 & 1 & 7 \\
\hline Classify & & 1 & & & & 1 & & & & & 2 \\
\hline Comparing & & 1 & & & 1 & 1 & 1 & 1 & 1 & 1 & 7 \\
\hline Application & & & & & & & & & & & \\
\hline
\end{tabular}


Development, Item Analysis and Standardization of Teachers Cognitive Ability Test

\begin{tabular}{|l|c|c|c|c|c|c|c|c|c|c|c|}
\hline Solve & & & 1 & 1 & & & & & & 1 & 3 \\
\hline Relate & 1 & 1 & & & 1 & & 1 & 1 & 1 & & 6 \\
\hline Analysis & & & & & & & & & & & \\
\hline Analyse & 1 & 1 & 1 & 1 & 1 & & 1 & 1 & & 1 & 8 \\
\hline Discriminate & & & & & 1 & 1 & & 1 & 1 & & 4 \\
\hline Synthesis & & & & & & & & & & & \\
\hline Devise & 1 & 1 & 1 & 1 & 1 & 1 & & 1 & 1 & 1 & 9 \\
\hline Evaluation & & & & & & & & & & & \\
\hline Justify & 1 & 1 & 1 & 1 & 1 & 1 & 1 & 1 & 1 & 1 & 10 \\
\hline & & & & & & & & & & & \\
\hline TOTAL & $\mathbf{5}$ & $\mathbf{8}$ & $\mathbf{6}$ & $\mathbf{6}$ & $\mathbf{8}$ & $\mathbf{7}$ & $\mathbf{6}$ & $\mathbf{7}$ & $\mathbf{7}$ & $\mathbf{8}$ & \\
\hline
\end{tabular}

\begin{tabular}{|c|c|c|c|c|c|c|c|c|c|c|c|}
\hline & Item & Item & Item & Item & Item & Item & Item & Item & Item & Item & TOTAL \\
\hline \begin{tabular}{|l} 
Level of \\
Learning \\
Outcomes \\
\end{tabular} & 61 & 62 & 63 & 64 & 65 & 66 & 67 & 68 & 69 & 70 & \\
\hline \multicolumn{12}{|l|}{ Knowledge } \\
\hline Recall & 1 & 1 & 1 & 1 & & 1 & & & & & 5 \\
\hline Identify & 1 & & & & 1 & 1 & 1 & 1 & 1 & 1 & 7 \\
\hline \multicolumn{12}{|c|}{ Comprehension } \\
\hline Interpret & 1 & & & & 1 & & 1 & 1 & 1 & & 5 \\
\hline Classify & & 1 & & & & & & & & & 1 \\
\hline Comparing & 1 & & 1 & 1 & & 1 & 1 & & & 1 & 6 \\
\hline \multicolumn{12}{|l|}{ Application } \\
\hline Solve & 1 & & & & & & & & 1 & & 2 \\
\hline Relate & & 1 & 1 & 1 & 1 & 1 & 1 & 1 & & 1 & 8 \\
\hline \multicolumn{12}{|l|}{ Analysis } \\
\hline Analyse & 1 & & 1 & 1 & 1 & & 1 & 1 & 1 & & 7 \\
\hline Discriminate & & 1 & & & & 1 & & & & 1 & 3 \\
\hline \multicolumn{12}{|l|}{ Synthesis } \\
\hline Devise & 1 & 1 & 1 & 1 & 1 & & & 1 & 1 & 1 & 8 \\
\hline \multicolumn{12}{|l|}{ Evaluation } \\
\hline Justify & 1 & 1 & 1 & 1 & 1 & 1 & 1 & 1 & 1 & 1 & 10 \\
\hline TOTAL & 8 & 6 & 6 & 6 & 6 & 6 & 6 & 6 & 6 & 5 & \\
\hline
\end{tabular}


Development, Item Analysis and Standardization of Teachers Cognitive Ability Test

\begin{tabular}{|c|c|c|c|c|c|c|c|c|c|c|c|}
\hline & Item & Item & Item & Item & Item & Item & Item & Item & Item & Item & TOTAL \\
\hline $\begin{array}{l}\text { Level of } \\
\text { Learning } \\
\text { Outcomes }\end{array}$ & 71 & 72 & 73 & 74 & 75 & 76 & 77 & 78 & 79 & 80 & \\
\hline \multicolumn{12}{|l|}{ Knowledge } \\
\hline Recall & 1 & 1 & 1 & 1 & 1 & 1 & 1 & 1 & 1 & 1 & 10 \\
\hline Identify & 1 & 1 & 1 & 1 & & & & & & & 4 \\
\hline \multicolumn{12}{|c|}{ Comprehension } \\
\hline Interpret & & & & & 1 & 1 & 1 & 1 & 1 & 1 & 6 \\
\hline Classify & 1 & 1 & 1 & 1 & & & & & & & 4 \\
\hline Comparing & 1 & 1 & 1 & 1 & 1 & 1 & 1 & 1 & 1 & 1 & 10 \\
\hline \multicolumn{12}{|l|}{ Application } \\
\hline Solve & & & & & 1 & 1 & 1 & 1 & 1 & 1 & 6 \\
\hline Relate & & & & & & & & & & & 0 \\
\hline \multicolumn{12}{|l|}{ Analysis } \\
\hline Analyse & & & & & 1 & 1 & 1 & 1 & 1 & 1 & 6 \\
\hline Discriminate & 1 & 1 & 1 & 1 & 1 & 1 & 1 & 1 & 1 & 1 & 10 \\
\hline \multicolumn{12}{|l|}{ Synthesis } \\
\hline Devise & 1 & 1 & 1 & 1 & 1 & 1 & 1 & 1 & 1 & 1 & 10 \\
\hline \multicolumn{12}{|l|}{ Evaluation } \\
\hline \begin{tabular}{|l|} 
Justify \\
\end{tabular} & 1 & 1 & 1 & 1 & 1 & 1 & 1 & 1 & 1 & 1 & 10 \\
\hline TOTAL & 7 & 7 & 7 & 7 & 8 & 8 & 8 & 8 & 8 & 8 & \\
\hline
\end{tabular}

Table 4: Summary of item analysis

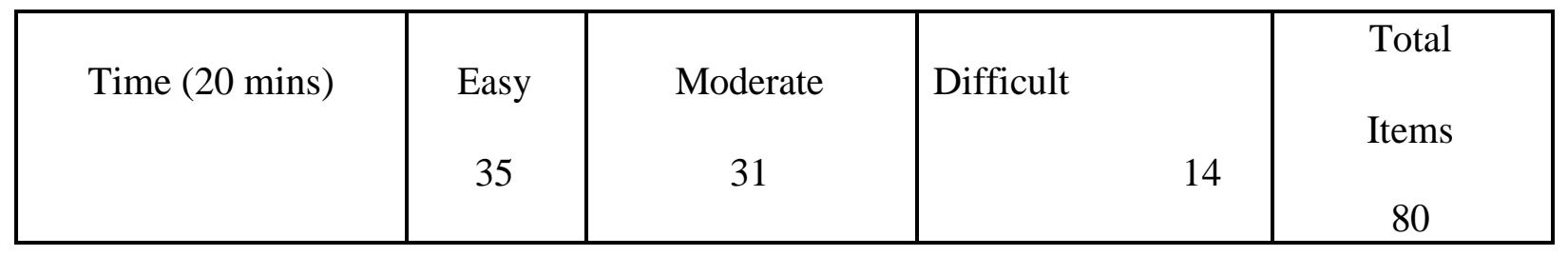

An Item with 50\% difficulty, level is considered to be an ideal test item. However research shows that items with discriminations indices ranging from $16 \%$ to $84 \%$ could be included preferably. To this item analysis researches followed these criteria. However some expert of the field such as Ebel and Frisbie (1986, P. 324) also accept it as valid beyond this range. But in no case items with discrimination indices less than or equal to zero were accepted. 
Total No. of Items in Test $=80$

$$
\text { Item difficulty index }=P=\frac{V_{P}}{V}
$$

$\mathrm{NP}$ - Indicates the number of test takers in total group who passed the item $=17$

$\mathrm{N}$ - Indicates the total number of test takers in the group $=383$

The item difficulty index (P) has a range of 0.00 to 1.00. If no one answers the item correctly, $\mathrm{P}$ value would be 0.00 . An item that everyone answer correctly would have a $\mathrm{P}$ value of 1.00

Item: Discrimination Index is $D=\frac{U_{p}-L_{p}}{U}$

UP - No. of test takers in upper group LP - No. of test takers in lower group

$\mathrm{U}-$ is the total number of test takers in upper group

The optional level for an acceptable P value depends on the no. of options per item. In present test, have 4 options Then $\mathrm{g}=.25$

$\mathrm{P}$ value $=1.0 \mathrm{G}$ value $=.25$

Constant value $=2$

$\underline{1.0+.25}$

2

Optional level $=.63$

As the number of options increases, the option $\mathrm{P}$ - value decreases, these test have more option to also be more difficult to answer.

The difficulty level increases.

After optional level of item: - we get lower Bond

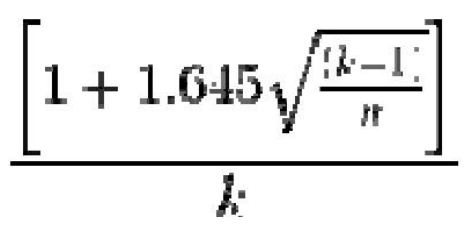

$\mathrm{K}=$ No. of multiple choice item $\mathrm{K}=80$

$\mathrm{N}-$ No. of examiners $\mathrm{N}=383$ 
Table 5: items with difficulty level $<16$

\begin{tabular}{|l|l|}
\hline Items & $10,14,15,16,18,19,20,28,38,39,40,41,42,48,49,50,5152$, \\
& $53,54,55,56,57,58,59,60,61,62,63,64,65,66,68,69,70,72$ \\
& $, 73,74,71,67,75,76,77,78,79,80$ \\
\hline
\end{tabular}

Difficulty level $<16$ means these items are very difficult.

Table 6: of items with difficulty level $>84$

\begin{tabular}{|l|l|}
\hline Items & $1,2,3,4,5,6,7,8,9,11,12,13,17,21,22,23,24,25,26,27,29,3$ \\
& $0,31,32,33,34,35,36,37,43,44,45,46,47$, \\
\hline
\end{tabular}

Difficulty level $>84$ means that items are very easy because the \%age of both high achieve and low achieve is high in these items.

Table 7: Numbers of items with index of discriminations

\begin{tabular}{|l|l|}
\hline Items & 7,8,15,16,18,19,20,22,23,28,44,45,52,54,55,56,57,58,5 \\
& $9,60,61,62,68,69,70,73,74,71,67,75,76,77,78,79,80$ \\
\hline
\end{tabular}

Ebel\&Frisbie (1986) gives us the following role of thumb for determining the quality of the items in terms of the discrimination index

Table 8: Shows the value $D$ and their corresponding interpretation

\begin{tabular}{|c|c|c|}
\hline D & Quality & Recommendation \\
\hline$>0.39$ & Excellent & Retain \\
\hline $0.30-0.39$ & Good & Possibilities for improvement \\
\hline $0.20-0.29$ & Mediocre & Need to check/review \\
\hline $0.00-0.20$ & Poor & Discard or reviewing depth \\
\hline$<-0.01$ & Worst & Definitely Discard \\
\hline
\end{tabular}

Pearson Correlation Coefficient (r) is used for measuring the linear dependence of two variables.

Pearson Correlation Coefficient Formula:

$$
r=\frac{n \sum_{i=1}^{n} x_{i} y_{i}-\sum_{i=1}^{n} x_{i} \sum_{i=1}^{n} y_{i}}{\sqrt{\left(n \sum_{i=1}^{n} x_{i}^{2}-\left(\sum_{i=1}^{n} x_{i}\right)^{2}\right)\left(n \sum_{i=1}^{n} y_{i}^{2}-\left(\sum_{i=1}^{n} y_{i}\right)^{2}\right)}}
$$


Where:

- $\quad \mathbf{x}_{\mathbf{i}}$ : the ith number of $\mathrm{x}$

- $\mathbf{y}_{\mathbf{i}}$ : the ith number of $\mathrm{y}$

- $\quad$ : total numbers of $\mathrm{x}$ or $\mathrm{y}$

$\square$ : correlation coefficient, $-1<=\mathrm{r}<=1,1$ represents strongly positively correlated, -1 represents strongly negatively correlated, 0 represents no correlation.

Table 9 Analysis of Reliability

$\mathrm{N}=383$

\begin{tabular}{|l|l|l|}
\hline Reliability & Correlation coefficient & Inference \\
\hline Test-Retest Method & 0.931843 & Highly significant \\
\hline
\end{tabular}

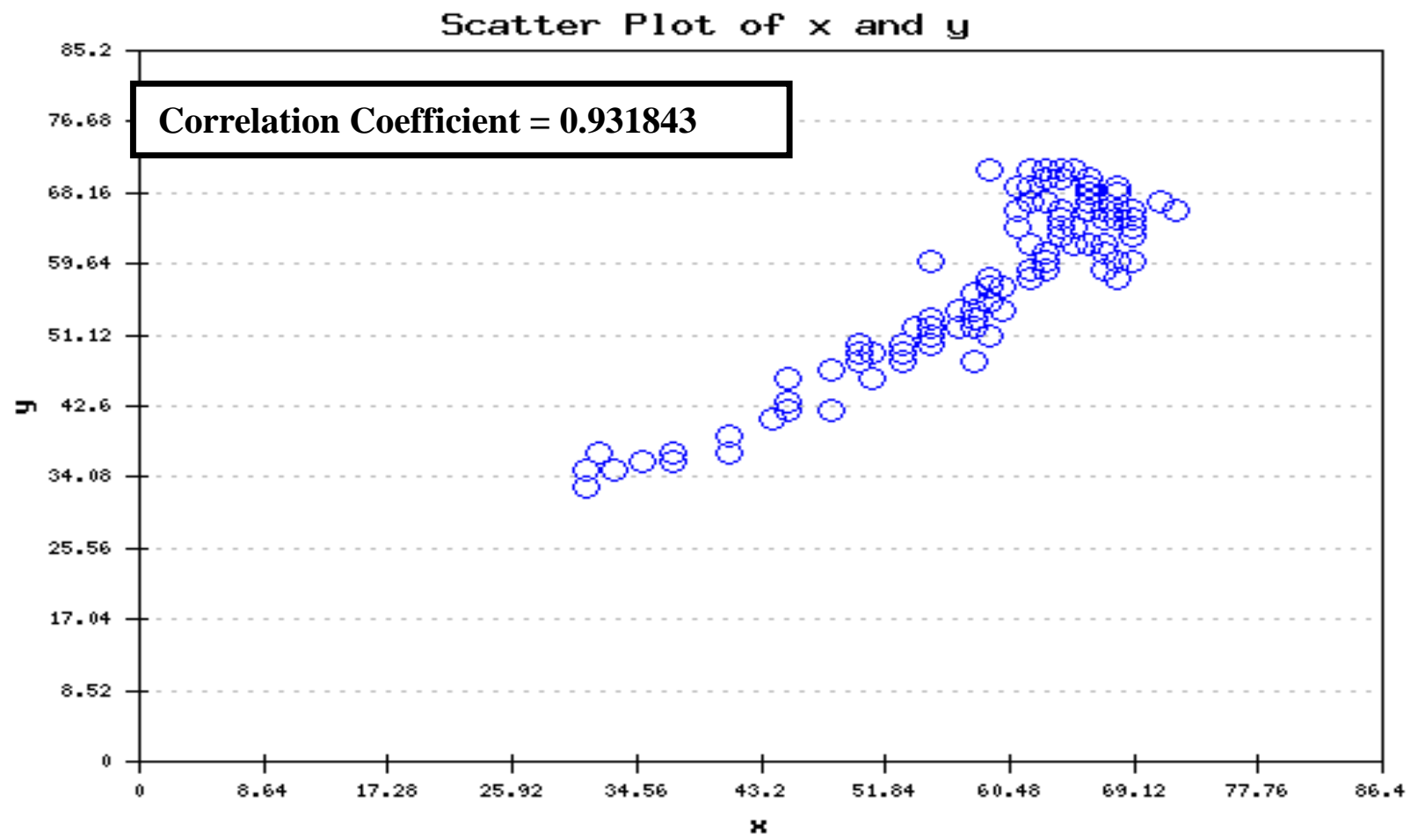

Scores of subjects in Test1

Fig.2 Correlation coefficient of the scores of Test1 and Test2 $(n=383)$ 
Development, Item Analysis and Standardization of Teachers Cognitive Ability Test

Table 10 Estimating Validity through Linear Regression Method

\begin{tabular}{|c|c|c|c|c|c|c|c|c|c|c|c|}
\hline S.No & $\begin{array}{r}\mathbf{Y} \\
\text { (criter } \\
\text { ion) } \\
\end{array}$ & $\begin{array}{r}\mathrm{X} \\
\text { (pract } \\
\text { ical) } \\
\end{array}$ & $\begin{array}{r}\text { pre } \\
\text { dict } \\
\text { ed }\end{array}$ & $\begin{array}{r}\text { resid } \\
\text { ual } \\
\end{array}$ & $\begin{array}{r}\text { prac- } \\
\text { crit } \\
\end{array}$ & S.no & $\begin{array}{l}1001 \\
\text { ogY } \\
\end{array}$ & $\begin{array}{r}100 \log \\
\mathrm{X}\end{array}$ & $\begin{array}{r}\text { predi } \\
\text { cted } \\
\end{array}$ & $\begin{array}{r}\text { resid } \\
\text { ual } \\
\end{array}$ & $\begin{array}{r}\text { prac- } \\
\text { crit } \\
\end{array}$ \\
\hline 1. & 51 & 55 & 52.52 & -1.52 & 4.00 & 1. & 393.2 & 400.7 & 396.23 & -3.05 & 7.55 \\
\hline 2. & 52 & 55 & 52.52 & -0.52 & 3.00 & 2. & 395.1 & 400.7 & 396.23 & -1.11 & 5.61 \\
\hline 3. & 54 & 58 & 55.18 & -1.18 & 4.00 & 3. & 398.9 & 406.0 & 400.98 & -2.08 & 7.15 \\
\hline 4. & 53 & 58 & 55.18 & -2.18 & 5.00 & 4. & 397.0 & 406.0 & 400.98 & -3.95 & 9.02 \\
\hline 5. & 53 & 58 & 55.18 & -2.18 & 5.00 & 5. & 397.0 & 406.0 & 400.98 & -3.95 & 9.02 \\
\hline 6. & 56 & 58 & 55.18 & 0.82 & 2.00 & 6. & 402.5 & 406.0 & 400.98 & 1.56 & 3.51 \\
\hline 7. & 58 & 59 & 56.06 & 1.94 & 1.00 & 7. & 406.0 & 407.8 & 402.51 & 3.54 & 1.71 \\
\hline 8. & 54 & 60 & 56.95 & -2.95 & 6.00 & 8. & 398.9 & 409.4 & 404.01 & -5.11 & 10.54 \\
\hline 9. & 57 & 60 & 56.95 & 0.05 & 3.00 & 9. & 404.3 & 409.4 & 404.01 & 0.29 & 5.13 \\
\hline 10. & 59 & 62 & 58.72 & 0.28 & 3.00 & 10. & $\begin{array}{l}407.8 \\
\end{array}$ & 412.7 & 406.94 & 0.81 & 4.96 \\
\hline 11. & 60 & 63 & 59.60 & 0.40 & 3.00 & 11. & 409.4 & 414.3 & 408.37 & 1.06 & 4.88 \\
\hline 12. & 59 & 67 & 63.14 & -4.14 & 8.00 & 12. & 407.8 & 420.5 & 413.88 & -6.12 & 12.72 \\
\hline 13. & 58 & 68 & 64.03 & -6.03 & 10.00 & 13. & 406.0 & 422.0 & 415.20 & -9.16 & 15.91 \\
\hline 14. & 66 & 69 & 64.91 & 1.09 & 3.00 & 14. & 419.0 & 423.4 & 416.51 & 2.46 & 4.45 \\
\hline 15. & 55 & 59 & 56.06 & -1.06 & 4.00 & 15. & 400.7 & 407.8 & 402.51 & -1.77 & 7.02 \\
\hline 16. & 62 & 66 & 62.26 & -0.26 & 4.00 & 16. & 412.7 & 419.0 & 412.53 & 0.18 & 6.25 \\
\hline 17. & 60 & 69 & 64.91 & -4.91 & 9.00 & 17. & 409.4 & 423.4 & 416.51 & -7.07 & 13.98 \\
\hline 18. & 65 & 69 & 64.91 & 0.09 & 4.00 & 18. & 417.4 & 423.4 & 416.51 & 0.93 & 5.97 \\
\hline 19. & 64 & 69 & 64.91 & -0.91 & 5.00 & 19. & 415.9 & 423.4 & 416.51 & -0.62 & 7.52 \\
\hline 20. & 64 & 61 & 57.83 & 6.17 & -3.00 & 20. & 415.9 & 411.1 & 405.49 & 10.40 & -4.80 \\
\hline 21. & 54 & 57 & 54.29 & -0.29 & 3.00 & 21. & 398.9 & 404.3 & 399.42 & -0.53 & 5.41 \\
\hline 22. & 59 & 63 & 59.60 & -0.60 & 4.00 & 22. & 407.8 & 414.3 & 408.37 & -0.62 & 6.56 \\
\hline 23. & 64 & 69 & 64.91 & -0.91 & 5.00 & 23. & 415.9 & 423.4 & 416.51 & -0.62 & 7.52 \\
\hline 24. & 62 & 65 & 61.37 & 0.63 & 3.00 & 24. & 412.7 & 417.4 & 411.17 & 1.55 & 4.73 \\
\hline 25. & 37 & 32 & 32.17 & 4.83 & -5.00 & 25. & 361.1 & 346.6 & 347.80 & 13.29 & -14.52 \\
\hline 26. & 36 & 37 & 36.59 & -0.59 & 1.00 & 26. & 358.4 & 361.1 & 360.78 & -2.43 & 2.74 \\
\hline 27. & 42 & 45 & 43.67 & -1.67 & 3.00 & 27. & $\begin{array}{l}373.8 \\
\end{array}$ & 380.7 & 378.29 & -4.52 & 6.90 \\
\hline 28. & 49 & 51 & 48.98 & 0.02 & 2.00 & 28. & 389.2 & 393.2 & 389.48 & -0.30 & 4.00 \\
\hline 29. & 55 & 59 & 56.06 & -1.06 & 4.00 & 29. & 400.7 & 407.8 & 402.51 & -1.77 & 7.02 \\
\hline 30. & 57 & 60 & 56.95 & 0.05 & 3.00 & 30. & 404.3 & 409.4 & 404.01 & 0.29 & 5.13 \\
\hline 31. & 65 & 64 & 60.49 & 4.51 & -1.00 & 31. & 417.4 & 415.9 & 409.78 & 7.66 & -1.55 \\
\hline 32. & 60 & 68 & 64.03 & -4.03 & 8.00 & 32. & 409.4 & 422.0 & 415.20 & -5.77 & 12.52 \\
\hline 33. & 68 & 68 & 64.03 & 3.97 & 0.00 & 33. & 422.0 & 422.0 & 415.20 & 6.75 & 0.00 \\
\hline 34. & 64 & 64 & 60.49 & 3.51 & 0.00 & 34. & 415.9 & 415.9 & 409.78 & 6.11 & 0.00 \\
\hline 35. & 66 & 68 & 64.03 & 1.97 & 2.00 & 35. & 419.0 & 422.0 & 415.20 & 3.76 & 2.99 \\
\hline 36. & 66 & 69 & 64.91 & 1.09 & 3.00 & 36. & 419.0 & 423.4 & 416.51 & 2.46 & 4.45 \\
\hline 37. & 66 & 68 & 64.03 & 1.97 & 2.00 & 37. & 419.0 & 422.0 & 415.20 & 3.76 & 2.99 \\
\hline 38. & 67 & 62 & 58.72 & 8.28 & -5.00 & 38. & 420.5 & 412.7 & 406.94 & 13.53 & -7.76 \\
\hline 39. & 51 & 59 & 56.06 & -5.06 & 8.00 & 39. & 393.2 & 407.8 & 402.51 & -9.32 & 14.57 \\
\hline 40. & 63 & 69 & 64.91 & -1.91 & 6.00 & 40. & 414.3 & 423.4 & 416.51 & -2.19 & 9.10 \\
\hline 41. & 66 & 61 & 57.83 & 8.17 & -5.00 & 41. & 419.0 & 411.1 & 405.49 & 13.48 & -7.88 \\
\hline 42. & 64 & 65 & 61.37 & 2.63 & 1.00 & 42. & 415.9 & 417.4 & 411.17 & 4.72 & 1.55 \\
\hline 43. & 69 & 66 & 62.26 & 6.74 & -3.00 & 43. & 423.4 & 419.0 & 412.53 & 10.88 & -4.45 \\
\hline 44. & 57 & 59 & 56.06 & 0.94 & 2.00 & 44. & 404.3 & 407.8 & 402.51 & 1.80 & 3.45 \\
\hline
\end{tabular}


Development, Item Analysis and Standardization of Teachers Cognitive Ability Test

\begin{tabular}{|c|c|c|c|c|c|c|c|c|c|c|c|}
\hline 45. & 69 & 66 & 62.26 & 6.74 & -3.00 & 45. & 423.4 & 419.0 & 412.53 & 10.88 & -4.45 \\
\hline 46. & 58 & 62 & 58.72 & -0.72 & 4.00 & 46. & 406.0 & 412.7 & 406.94 & -0.90 & 6.67 \\
\hline 47. & 52 & 54 & 51.64 & 0.36 & 2.00 & 47. & 395.1 & 398.9 & 394.59 & 0.53 & 3.77 \\
\hline 48. & 46 & 51 & $\begin{array}{l}48.98 \\
\end{array}$ & $\begin{array}{r}-2.98 \\
\end{array}$ & 5.00 & 48. & 382.9 & 393.2 & $\begin{array}{l}389.48 \\
\end{array}$ & -6.61 & 10.32 \\
\hline 49. & 49 & 50 & 48.10 & 0.90 & 1.00 & 49. & 389.2 & 391.2 & 387.71 & 1.47 & 2.02 \\
\hline 50. & 65 & 64 & 60.49 & 4.51 & -1.00 & 50. & 417.4 & 415.9 & 409.78 & 7.66 & -1.55 \\
\hline 51. & 39 & 41 & 40.13 & -1.13 & 2.00 & 51. & 366.4 & 371.4 & 369.96 & -3.61 & 5.00 \\
\hline 52. & 52 & 57 & 54.29 & -2.29 & 5.00 & 52. & 395.1 & 404.3 & 399.42 & -4.30 & 9.18 \\
\hline 53. & 71 & 59 & 56.06 & 14.94 & -12.00 & 53. & 426.3 & 407.8 & 402.51 & 23.76 & -18.51 \\
\hline 54. & 70 & 63 & 59.60 & 10.40 & -7.00 & 54. & 424.8 & 414.3 & 408.37 & 16.48 & -10.54 \\
\hline 55. & 37 & 41 & 40.13 & -3.13 & 4.00 & 55. & 361.1 & 371.4 & 369.96 & -8.87 & 10.27 \\
\hline 56. & 64 & 69 & 64.91 & -0.91 & 5.00 & 56. & 415.9 & 423.4 & 416.51 & -0.62 & 7.52 \\
\hline 57. & 61 & 63 & 59.60 & 1.40 & 2.00 & 57. & 411.1 & 414.3 & 408.37 & 2.71 & 3.23 \\
\hline 58. & 54 & 57 & 54.29 & -0.29 & 3.00 & 58. & 398.9 & 404.3 & 399.42 & -0.53 & 5.41 \\
\hline 59. & 59 & 63 & 59.60 & -0.60 & 4.00 & 59. & 407.8 & 414.3 & 408.37 & -0.62 & 6.56 \\
\hline 60. & 64 & 69 & 64.91 & -0.91 & 5.00 & 60. & 415.9 & 423.4 & 416.51 & -0.62 & 7.52 \\
\hline 61. & 62 & 65 & 61.37 & 0.63 & 3.00 & 61. & 412.7 & 417.4 & 411.17 & 1.55 & 4.73 \\
\hline 62. & 37 & 32 & 32.17 & 4.83 & -5.00 & 62. & 361.1 & 346.6 & 347.80 & 13.29 & -14.52 \\
\hline 63. & 36 & 37 & 36.59 & -0.59 & 1.00 & 63. & 358.4 & 361.1 & 360.78 & -2.43 & 2.74 \\
\hline 64. & 42 & 45 & 43.67 & -1.67 & 3.00 & 64. & 373.8 & 380.7 & 378.29 & -4.52 & 6.90 \\
\hline 65. & 49 & 51 & 48.98 & 0.02 & 2.00 & 65. & 389.2 & 393.2 & 389.48 & -0.30 & 4.00 \\
\hline 66. & 52 & 58 & 55.18 & -3.18 & 6.00 & 66. & 395.1 & 406.0 & 400.98 & -5.85 & 10.92 \\
\hline 67. & 48 & 58 & 55.18 & -7.18 & 10.00 & 67. & 387.1 & 406.0 & 400.98 & -13.86 & 18.92 \\
\hline 68. & 59 & 63 & 59.60 & -0.60 & 4.00 & 68. & 407.8 & 414.3 & 408.37 & -0.62 & 6.56 \\
\hline 69. & 47 & 48 & 46.33 & 0.67 & 1.00 & 69. & 385.0 & 387.1 & 384.06 & 0.96 & 2.11 \\
\hline 70. & 41 & 44 & 42.79 & -1.79 & 3.00 & 70. & 371.4 & 378.4 & 376.28 & -4.92 & 7.06 \\
\hline 71. & 50 & 55 & 52.52 & -2.52 & 5.00 & 71. & 391.2 & 400.7 & 396.23 & -5.03 & 9.53 \\
\hline 72. & 55 & 59 & 56.06 & -1.06 & 4.00 & 72. & 400.7 & 407.8 & 402.51 & -1.77 & 7.02 \\
\hline 73. & 50 & 55 & 52.52 & -2.52 & 5.00 & 73. & 391.2 & 400.7 & 396.23 & -5.03 & 9.53 \\
\hline 74. & 54 & 58 & 55.18 & -1.18 & 4.00 & 74. & 398.9 & 406.0 & 400.98 & -2.08 & 7.15 \\
\hline 75. & 66 & 72 & 67.57 & -1.57 & 6.00 & 75. & 419.0 & 427.7 & 420.31 & -1.35 & 8.70 \\
\hline 76. & 36 & 35 & 34.82 & 1.18 & -1.00 & 76. & 358.4 & 355.5 & 355.82 & 2.54 & -2.82 \\
\hline 77. & 48 & 50 & 48.10 & -0.10 & 2.00 & 77. & 387.1 & 391.2 & 387.71 & -0.59 & 4.08 \\
\hline 78. & 58 & 62 & 58.72 & -0.72 & 4.00 & 78. & 406.0 & 412.7 & 406.94 & -0.90 & 6.67 \\
\hline 79. & 52 & 54 & 51.64 & 0.36 & 2.00 & 79. & 395.1 & 398.9 & 394.59 & 0.53 & 3.77 \\
\hline 80. & 46 & 51 & 48.98 & -2.98 & 5.00 & 80. & 382.9 & 393.2 & 389.48 & -6.61 & 10.32 \\
\hline 81. & 49 & 50 & 48.10 & 0.90 & 1.00 & 81. & 389.2 & 391.2 & 387.71 & 1.47 & 2.02 \\
\hline 82. & 65 & 64 & 60.49 & 4.51 & -1.00 & 82. & 417.4 & 415.9 & 409.78 & 7.66 & -1.55 \\
\hline 83. & 39 & 41 & 40.13 & -1.13 & 2.00 & 83. & 366.4 & 371.4 & 369.96 & -3.61 & 5.00 \\
\hline 84. & 52 & 57 & 54.29 & -2.29 & 5.00 & 84. & 395.1 & 404.3 & 399.42 & -4.30 & 9.18 \\
\hline 85. & 71 & 63 & 59.60 & 11.40 & -8.00 & 85. & 426.3 & 414.3 & 408.37 & 17.90 & -11.95 \\
\hline 86. & 70 & 66 & 62.26 & 7.74 & -4.00 & 86. & 424.8 & 419.0 & 412.53 & 12.32 & -5.88 \\
\hline 87. & 37 & 41 & 40.13 & -3.13 & 4.00 & 87. & 361.1 & 371.4 & 369.96 & -8.87 & 10.27 \\
\hline 88. & 46 & 45 & 43.67 & 2.33 & -1.00 & 88. & 382.9 & 380.7 & 378.29 & 4.58 & -2.20 \\
\hline 89. & 60 & 55 & 52.52 & 7.48 & -5.00 & 89. & 409.4 & 400.7 & 396.23 & 13.20 & -8.70 \\
\hline 90. & 67 & 66 & 62.26 & 4.74 & -1.00 & 90. & 420.5 & 419.0 & 412.53 & 7.94 & -1.50 \\
\hline 91. & 37 & 37 & 36.59 & 0.41 & 0.00 & 91. & 361.1 & 361.1 & 360.78 & 0.31 & 0.00 \\
\hline 92. & 53 & 55 & 52.52 & 0.48 & 2.00 & 92. & 397.0 & 400.7 & 396.23 & 0.80 & 3.70 \\
\hline 93. & 62 & 67 & 63.14 & -1.14 & 5.00 & 93. & 412.7 & 420.5 & 413.88 & -1.16 & 7.76 \\
\hline
\end{tabular}


Development, Item Analysis and Standardization of Teachers Cognitive Ability Test

\begin{tabular}{|c|c|c|c|c|c|c|c|c|c|c|c|}
\hline 94. & 67 & 71 & 66.68 & 0.32 & 4.00 & 94. & 420.5 & 426.3 & 419.06 & 1.41 & 5.80 \\
\hline 95. & 69 & 61 & 57.83 & 11.17 & -8.00 & 95. & 423.4 & 411.1 & 405.49 & 17.92 & -12.32 \\
\hline 96. & 66 & 64 & 60.49 & 5.51 & -2.00 & 96. & 419.0 & 415.9 & 409.78 & 9.18 & -3.08 \\
\hline 97. & 65 & 67 & 63.14 & 1.86 & 2.00 & 97. & 417.4 & 420.5 & 413.88 & 3.56 & 3.03 \\
\hline 98. & 69 & 68 & 64.03 & 4.97 & -1.00 & 98. & 423.4 & 422.0 & 415.20 & 8.21 & -1.46 \\
\hline 99. & 65 & 68 & 64.03 & 0.97 & 3.00 & 99. & 417.4 & 422.0 & 415.20 & 2.24 & 4.51 \\
\hline 100. & 63 & 64 & 60.49 & 2.51 & 1.00 & 100. & 414.3 & 415.9 & 409.78 & 4.53 & 1.57 \\
\hline 101. & 33 & 31 & 31.28 & 1.72 & -2.00 & 101. & 349.7 & 343.4 & 344.96 & 4.69 & -6.25 \\
\hline 102. & 35 & 31 & 31.28 & 3.72 & -4.00 & 102. & 355.5 & 343.4 & 344.96 & 10.57 & -12.14 \\
\hline 103. & 50 & 53 & 50.75 & -0.75 & 3.00 & 103. & 391.2 & 397.0 & 392.92 & -1.72 & 5.83 \\
\hline 104. & 51 & 55 & 52.52 & -1.52 & 4.00 & 104. & 393.2 & 400.7 & 396.23 & -3.05 & 7.55 \\
\hline 105. & 52 & 55 & 52.52 & -0.52 & 3.00 & 105. & 395.1 & 400.7 & 396.23 & -1.11 & 5.61 \\
\hline 106. & 54 & 58 & 55.18 & -1.18 & 4.00 & 106. & 398.9 & 406.0 & 400.98 & -2.08 & 7.15 \\
\hline 107. & 53 & 58 & 55.18 & -2.18 & 5.00 & 107. & 397.0 & 406.0 & 400.98 & -3.95 & 9.02 \\
\hline 108. & 53 & 58 & 55.18 & -2.18 & 5.00 & 108. & 397.0 & 406.0 & 400.98 & -3.95 & 9.02 \\
\hline 109. & 56 & 58 & 55.18 & 0.82 & 2.00 & 109. & 402.5 & 406.0 & 400.98 & 1.56 & 3.51 \\
\hline 110. & 58 & 59 & 56.06 & 1.94 & 1.00 & 110. & 406.0 & 407.8 & 402.51 & 3.54 & 1.71 \\
\hline 111. & 54 & 60 & 56.95 & -2.95 & 6.00 & 111. & 398.9 & 409.4 & 404.01 & -5.11 & 10.54 \\
\hline 112. & 57 & 60 & 56.95 & 0.05 & 3.00 & 112. & 404.3 & 409.4 & 404.01 & 0.29 & 5.13 \\
\hline 113. & 59 & 62 & 58.72 & 0.28 & 3.00 & 113. & 407.8 & 412.7 & 406.94 & 0.81 & 4.96 \\
\hline 114. & 60 & 63 & 59.60 & 0.40 & 3.00 & 114. & 409.4 & 414.3 & 408.37 & 1.06 & 4.88 \\
\hline 115. & 59 & 67 & 63.14 & -4.14 & 8.00 & 115. & 407.8 & 420.5 & 413.88 & -6.12 & 12.72 \\
\hline 116. & 58 & 68 & 64.03 & -6.03 & 10.00 & 116. & 406.0 & 422.0 & 415.20 & -9.16 & 15.91 \\
\hline 117. & 66 & 69 & 64.91 & 1.09 & 3.00 & 117. & 419.0 & 423.4 & 416.51 & 2.46 & 4.45 \\
\hline 118. & 55 & 59 & 56.06 & -1.06 & 4.00 & 118. & 400.7 & 407.8 & 402.51 & -1.77 & 7.02 \\
\hline 119. & 62 & 66 & 62.26 & -0.26 & 4.00 & 119. & 412.7 & 419.0 & 412.53 & 0.18 & 6.25 \\
\hline 120. & 60 & 69 & 64.91 & -4.91 & 9.00 & 120. & 409.4 & 423.4 & 416.51 & -7.07 & 13.98 \\
\hline 121. & 50 & 55 & 52.52 & -2.52 & 5.00 & 121. & 391.2 & 400.7 & 396.23 & -5.03 & 9.53 \\
\hline 122. & 60 & 63 & 59.60 & 0.40 & 3.00 & 122. & 409.4 & 414.3 & 408.37 & 1.06 & 4.88 \\
\hline 123. & 47 & 48 & 46.33 & 0.67 & 1.00 & 123. & 385.0 & 387.1 & 384.06 & 0.96 & 2.11 \\
\hline 124. & 41 & 44 & 42.79 & -1.79 & 3.00 & 124. & 371.4 & 378.4 & 376.28 & -4.92 & 7.06 \\
\hline 125. & 50 & 55 & 52.52 & -2.52 & 5.00 & 125. & 391.2 & 400.7 & 396.23 & -5.03 & 9.53 \\
\hline 126. & 60 & 63 & 59.60 & 0.40 & 3.00 & 126. & 409.4 & 414.3 & 408.37 & 1.06 & 4.88 \\
\hline 127. & 62 & 67 & 63.14 & -1.14 & 5.00 & 127. & 412.7 & 420.5 & 413.88 & -1.16 & 7.76 \\
\hline 128. & 48 & 50 & 48.10 & -0.10 & 2.00 & 128. & 387.1 & 391.2 & 387.71 & -0.59 & 4.08 \\
\hline 129. & 50 & 50 & 48.10 & 1.90 & 0.00 & 129. & 391.2 & 391.2 & 387.71 & 3.49 & 0.00 \\
\hline 130. & 55 & 59 & 56.06 & -1.06 & 4.00 & 130. & 400.7 & 407.8 & 402.51 & -1.77 & 7.02 \\
\hline 131. & 50 & 55 & 52.52 & -2.52 & 5.00 & 131. & 391.2 & 400.7 & 396.23 & -5.03 & 9.53 \\
\hline 132. & 54 & 58 & 55.18 & -1.18 & 4.00 & 132. & 398.9 & 406.0 & 400.98 & -2.08 & 7.15 \\
\hline 133. & 66 & 72 & 67.57 & -1.57 & 6.00 & 133. & 419.0 & 427.7 & 420.31 & -1.35 & 8.70 \\
\hline 134. & 36 & 35 & 34.82 & 1.18 & -1.00 & 134. & 358.4 & 355.5 & 355.82 & 2.54 & -2.82 \\
\hline 135. & 48 & 50 & 48.10 & -0.10 & 2.00 & 135. & 387.1 & 391.2 & 387.71 & -0.59 & 4.08 \\
\hline 136. & 58 & 62 & 58.72 & -0.72 & 4.00 & 136. & 406.0 & 412.7 & 406.94 & -0.90 & 6.67 \\
\hline 137. & 52 & 54 & 51.64 & 0.36 & 2.00 & 137. & 395.1 & 398.9 & 394.59 & 0.53 & 3.77 \\
\hline 138. & 46 & 51 & $\begin{array}{r}48.98 \\
\end{array}$ & $\begin{array}{r}-2.98 \\
\end{array}$ & 5.00 & 138. & 382.9 & 393.2 & $\begin{array}{l}389.48 \\
\end{array}$ & -6.61 & 10.32 \\
\hline 139. & 49 & 50 & 48.10 & 0.90 & 1.00 & 139. & 389.2 & 391.2 & 387.71 & 1.47 & 2.02 \\
\hline 140. & 65 & 64 & 60.49 & 4.51 & -1.00 & 140. & 417.4 & 415.9 & 409.78 & 7.66 & -1.55 \\
\hline 141. & 39 & 41 & 40.13 & -1.13 & 2.00 & 141. & 366.4 & 371.4 & 369.96 & -3.61 & 5.00 \\
\hline 142. & 52 & 57 & 54.29 & -2.29 & 5.00 & 142. & 395.1 & 404.3 & 399.42 & -4.30 & 9.18 \\
\hline
\end{tabular}


Development, Item Analysis and Standardization of Teachers Cognitive Ability Test

\begin{tabular}{|c|c|c|c|c|c|c|c|c|c|c|c|}
\hline 143. & 71 & 64 & 60.49 & 10.51 & -7.00 & 143. & 426.3 & 415.9 & 409.78 & 16.49 & -10.38 \\
\hline 144. & 70 & 64 & 60.49 & 9.51 & -6.00 & 144. & 424.8 & 415.9 & 409.78 & 15.07 & -8.96 \\
\hline 145. & 37 & 41 & 40.13 & -3.13 & 4.00 & 145. & 361.1 & 371.4 & 369.96 & -8.87 & 10.27 \\
\hline 146. & 46 & 45 & 43.67 & 2.33 & -1.00 & 146. & 382.9 & 380.7 & 378.29 & 4.58 & -2.20 \\
\hline 147. & 43 & 45 & 43.67 & -0.67 & 2.00 & 147. & 376.1 & 380.7 & 378.29 & -2.17 & 4.55 \\
\hline 148. & 47 & 48 & 46.33 & 0.67 & 1.00 & 148. & 385.0 & 387.1 & 384.06 & 0.96 & 2.11 \\
\hline 149. & 42 & 48 & 46.33 & -4.33 & 6.00 & 149. & 373.8 & 387.1 & 384.06 & -10.29 & 13.35 \\
\hline 150. & 48 & 53 & 50.75 & -2.75 & 5.00 & 150. & 387.1 & 397.0 & 392.92 & -5.80 & 9.91 \\
\hline 151. & 49 & 53 & 50.75 & -1.75 & 4.00 & 151. & 389.2 & 397.0 & 392.92 & -3.74 & 7.85 \\
\hline 152. & 50 & 53 & 50.75 & -0.75 & 3.00 & 152. & 391.2 & 397.0 & 392.92 & -1.72 & 5.83 \\
\hline 153. & 49 & 50 & 48.10 & 0.90 & 1.00 & 153. & 389.2 & 391.2 & 387.71 & 1.47 & 2.02 \\
\hline 154. & 65 & 64 & 60.49 & 4.51 & -1.00 & 154. & 417.4 & 415.9 & 409.78 & 7.66 & -1.55 \\
\hline 155. & 66 & 69 & 64.91 & 1.09 & 3.00 & 155. & 419.0 & 423.4 & 416.51 & 2.46 & 4.45 \\
\hline 156. & 55 & 59 & 56.06 & -1.06 & 4.00 & 156. & 400.7 & 407.8 & 402.51 & -1.77 & 7.02 \\
\hline 157. & 62 & 66 & 62.26 & -0.26 & 4.00 & 157. & 412.7 & 419.0 & 412.53 & 0.18 & 6.25 \\
\hline 158. & 60 & 69 & 64.91 & -4.91 & 9.00 & 158. & 409.4 & 423.4 & 416.51 & -7.07 & 13.98 \\
\hline 159. & 65 & 69 & 64.91 & 0.09 & 4.00 & 159. & 417.4 & 423.4 & 416.51 & 0.93 & 5.97 \\
\hline 160. & 66 & 68 & 64.03 & 1.97 & 2.00 & 160. & 419.0 & 422.0 & 415.20 & 3.76 & 2.99 \\
\hline 161. & 66 & 61 & 57.83 & 8.17 & -5.00 & 161. & 419.0 & 411.1 & 405.49 & 13.48 & -7.88 \\
\hline 162. & 69 & 66 & 62.26 & 6.74 & -3.00 & 162. & 423.4 & 419.0 & 412.53 & 10.88 & -4.45 \\
\hline 163. & 57 & 59 & 56.06 & 0.94 & 2.00 & 163. & 404.3 & 407.8 & 402.51 & 1.80 & 3.45 \\
\hline 164. & 69 & 61 & 57.83 & 11.17 & -8.00 & 164. & 423.4 & 411.1 & 405.49 & 17.92 & -12.32 \\
\hline 165. & 65 & 67 & 63.14 & 1.86 & 2.00 & 165. & 417.4 & 420.5 & 413.88 & 3.56 & 3.03 \\
\hline 166. & 69 & 68 & 64.03 & 4.97 & -1.00 & 166. & 423.4 & 422.0 & 415.20 & 8.21 & -1.46 \\
\hline 167. & 65 & 68 & 64.03 & 0.97 & 3.00 & 167. & 417.4 & 422.0 & 415.20 & 2.24 & 4.51 \\
\hline 168. & 67 & 63 & 59.60 & 7.40 & -4.00 & 168. & 420.5 & 414.3 & 408.37 & 12.10 & -6.16 \\
\hline 169. & 33 & 31 & 31.28 & 1.72 & -2.00 & 169. & 349.7 & 343.4 & 344.96 & 4.69 & -6.25 \\
\hline 170. & 35 & 31 & 31.28 & 3.72 & -4.00 & 170. & 355.5 & 343.4 & 344.96 & 10.57 & -12.14 \\
\hline 171. & 50 & 53 & 50.75 & -0.75 & 3.00 & 171. & 391.2 & 397.0 & 392.92 & -1.72 & 5.83 \\
\hline 172. & 51 & 55 & 52.52 & -1.52 & 4.00 & 172. & 393.2 & 400.7 & 396.23 & -3.05 & 7.55 \\
\hline 173. & 52 & 55 & 52.52 & -0.52 & 3.00 & 173. & 395.1 & 400.7 & 396.23 & -1.11 & 5.61 \\
\hline 174. & 54 & 58 & 55.18 & -1.18 & 4.00 & 174. & 398.9 & 406.0 & 400.98 & -2.08 & 7.15 \\
\hline 175. & 53 & 58 & 55.18 & -2.18 & 5.00 & 175. & 397.0 & 406.0 & 400.98 & -3.95 & 9.02 \\
\hline 176. & 53 & 58 & 55.18 & -2.18 & 5.00 & 176. & 397.0 & 406.0 & 400.98 & -3.95 & 9.02 \\
\hline 177. & 56 & 58 & 55.18 & 0.82 & 2.00 & 177. & 402.5 & 406.0 & 400.98 & 1.56 & 3.51 \\
\hline 178. & 58 & 59 & 56.06 & 1.94 & 1.00 & 178. & 406.0 & 407.8 & 402.51 & 3.54 & 1.71 \\
\hline 179. & 54 & 60 & 56.95 & -2.95 & 6.00 & 179. & 398.9 & 409.4 & 404.01 & -5.11 & 10.54 \\
\hline 180. & 57 & 60 & 56.95 & 0.05 & 3.00 & 180. & 404.3 & 409.4 & 404.01 & 0.29 & 5.13 \\
\hline 181. & 59 & 62 & 58.72 & 0.28 & 3.00 & 181. & 407.8 & 412.7 & 406.94 & 0.81 & 4.96 \\
\hline 182. & 60 & 63 & 59.60 & 0.40 & 3.00 & 182. & 409.4 & 414.3 & 408.37 & 1.06 & 4.88 \\
\hline 183. & 59 & 67 & 63.14 & -4.14 & 8.00 & 183. & 407.8 & 420.5 & 413.88 & -6.12 & 12.72 \\
\hline 184. & 58 & 68 & 64.03 & -6.03 & 10.00 & 184. & 406.0 & 422.0 & 415.20 & -9.16 & 15.91 \\
\hline 185. & 66 & 69 & 64.91 & 1.09 & 3.00 & 185. & 419.0 & 423.4 & 416.51 & 2.46 & 4.45 \\
\hline 186. & 55 & 59 & 56.06 & -1.06 & 4.00 & 186. & 400.7 & 407.8 & 402.51 & -1.77 & 7.02 \\
\hline 187. & 62 & 66 & 62.26 & -0.26 & 4.00 & 187. & 412.7 & 419.0 & 412.53 & 0.18 & 6.25 \\
\hline 188. & 60 & 69 & 64.91 & -4.91 & 9.00 & 188. & 409.4 & 423.4 & 416.51 & -7.07 & 13.98 \\
\hline 189. & 50 & 55 & 52.52 & -2.52 & 5.00 & 189. & 391.2 & 400.7 & 396.23 & -5.03 & 9.53 \\
\hline 190. & 60 & 63 & 59.60 & 0.40 & 3.00 & 190. & 409.4 & 414.3 & 408.37 & 1.06 & 4.88 \\
\hline 191. & 62 & 67 & 63.14 & -1.14 & 5.00 & 191. & 412.7 & 420.5 & 413.88 & -1.16 & 7.76 \\
\hline
\end{tabular}


Development, Item Analysis and Standardization of Teachers Cognitive Ability Test

\begin{tabular}{|c|c|c|c|c|c|c|c|c|c|c|c|}
\hline 192. & 48 & 50 & 48.10 & -0.10 & 2.00 & 192. & 387.1 & 391.2 & 387.71 & -0.59 & 4.08 \\
\hline 193. & 50 & 50 & 48.10 & 1.90 & 0.00 & 193. & 391.2 & 391.2 & 387.71 & 3.49 & 0.00 \\
\hline 194. & 55 & 59 & 56.06 & -1.06 & 4.00 & 194. & 400.7 & 407.8 & 402.51 & -1.77 & 7.02 \\
\hline 195. & 50 & 55 & 52.52 & -2.52 & 5.00 & 195. & 391.2 & 400.7 & 396.23 & -5.03 & 9.53 \\
\hline 196. & 54 & 58 & 55.18 & -1.18 & 4.00 & 196. & 398.9 & 406.0 & 400.98 & -2.08 & 7.15 \\
\hline 197. & 66 & 72 & 67.57 & -1.57 & 6.00 & 197. & 419.0 & 427.7 & 420.31 & -1.35 & 8.70 \\
\hline 198. & 36 & 35 & 34.82 & 1.18 & -1.00 & 198. & 358.4 & 355.5 & 355.82 & 2.54 & -2.82 \\
\hline 199. & 48 & 50 & 48.10 & -0.10 & 2.00 & 199. & 387.1 & 391.2 & 387.71 & -0.59 & 4.08 \\
\hline 200. & 48 & 58 & 55.18 & -7.18 & 10.00 & 200. & 387.1 & 406.0 & 400.98 & -13.86 & 18.92 \\
\hline 201. & 59 & 63 & 59.60 & -0.60 & 4.00 & 201. & 407.8 & 414.3 & 408.37 & -0.62 & 6.56 \\
\hline 202. & 47 & 48 & 46.33 & 0.67 & 1.00 & 202. & 385.0 & 387.1 & 384.06 & 0.96 & 2.11 \\
\hline 203. & 41 & 44 & 42.79 & $\begin{array}{l}-1.79 \\
\end{array}$ & 3.00 & 203. & 371.4 & 378.4 & 376.28 & -4.92 & 7.06 \\
\hline 204. & 50 & 55 & 52.52 & -2.52 & 5.00 & 204. & 391.2 & 400.7 & 396.23 & -5.03 & 9.53 \\
\hline 205. & 60 & 63 & 59.60 & 0.40 & 3.00 & 205. & 409.4 & 414.3 & 408.37 & 1.06 & 4.88 \\
\hline 206. & 62 & 67 & 63.14 & -1.14 & 5.00 & 206. & 412.7 & 420.5 & 413.88 & -1.16 & 7.76 \\
\hline 207. & 48 & 50 & 48.10 & -0.10 & 2.00 & 207. & 387.1 & 391.2 & 387.71 & -0.59 & 4.08 \\
\hline 208. & 50 & 50 & 48.10 & 1.90 & 0.00 & 208. & 391.2 & 391.2 & 387.71 & 3.49 & 0.00 \\
\hline 209. & 55 & 59 & 56.06 & -1.06 & 4.00 & 209. & 400.7 & 407.8 & 402.51 & -1.77 & 7.02 \\
\hline 210. & 50 & 55 & 52.52 & -2.52 & 5.00 & 210. & 391.2 & 400.7 & 396.23 & -5.03 & 9.53 \\
\hline 211. & 54 & 58 & $\begin{array}{l}55.18 \\
\end{array}$ & -1.18 & 4.00 & 211. & 398.9 & 406.0 & 400.98 & -2.08 & 7.15 \\
\hline 212. & 66 & 72 & 67.57 & -1.57 & 6.00 & 212. & 419.0 & 427.7 & 420.31 & -1.35 & 8.70 \\
\hline 213. & 36 & 35 & 34.82 & 1.18 & -1.00 & 213. & 358.4 & 355.5 & 355.82 & 2.54 & -2.82 \\
\hline 214. & 48 & 50 & 48.10 & -0.10 & 2.00 & 214. & 387.1 & 391.2 & 387.71 & -0.59 & 4.08 \\
\hline 215. & 58 & 62 & 58.72 & -0.72 & 4.00 & 215. & 406.0 & 412.7 & 406.94 & -0.90 & 6.67 \\
\hline 216. & 52 & 54 & 51.64 & 0.36 & 2.00 & 216. & 395.1 & 398.9 & 394.59 & 0.53 & 3.77 \\
\hline 217. & 46 & 51 & 48.98 & -2.98 & 5.00 & 217. & 382.9 & 393.2 & 389.48 & -6.61 & 10.32 \\
\hline 218. & 53 & 58 & 55.18 & -2.18 & 5.00 & 218. & 397.0 & 406.0 & 400.98 & -3.95 & 9.02 \\
\hline 219. & 56 & 58 & 55.18 & 0.82 & 2.00 & 219. & 402.5 & 406.0 & 400.98 & 1.56 & 3.51 \\
\hline 220. & 58 & 59 & 56.06 & 1.94 & 1.00 & 220. & 406.0 & 407.8 & 402.51 & 3.54 & 1.71 \\
\hline 221. & 54 & 60 & 56.95 & -2.95 & 6.00 & 221. & 398.9 & 409.4 & 404.01 & -5.11 & 10.54 \\
\hline 222. & 57 & 60 & 56.95 & 0.05 & 3.00 & 222. & 404.3 & 409.4 & 404.01 & 0.29 & 5.13 \\
\hline 223. & 59 & 62 & 58.72 & 0.28 & 3.00 & 223. & 407.8 & 412.7 & 406.94 & 0.81 & 4.96 \\
\hline 224. & 60 & 63 & 59.60 & 0.40 & 3.00 & 224. & 409.4 & 414.3 & 408.37 & 1.06 & 4.88 \\
\hline 225. & 59 & 67 & 63.14 & -4.14 & 8.00 & 225. & 407.8 & 420.5 & 413.88 & -6.12 & 12.72 \\
\hline 226. & 58 & 68 & 64.03 & -6.03 & 10.00 & 226. & 406.0 & 422.0 & 415.20 & $\begin{array}{l}-9.16 \\
\end{array}$ & 15.91 \\
\hline 227. & 66 & 69 & 64.91 & 1.09 & 3.00 & 227. & 419.0 & 423.4 & 416.51 & 2.46 & 4.45 \\
\hline 228. & 55 & 59 & 56.06 & -1.06 & 4.00 & 228. & 400.7 & 407.8 & 402.51 & -1.77 & 7.02 \\
\hline 229. & 62 & 66 & 62.26 & -0.26 & 4.00 & 229. & 412.7 & 419.0 & 412.53 & 0.18 & 6.25 \\
\hline 230. & 60 & 69 & 64.91 & -4.91 & 9.00 & 230. & 409.4 & 423.4 & 416.51 & -7.07 & 13.98 \\
\hline 231. & 65 & 69 & 64.91 & 0.09 & 4.00 & 231. & 417.4 & 423.4 & 416.51 & 0.93 & 5.97 \\
\hline 232. & 64 & 69 & 64.91 & -0.91 & 5.00 & 232. & 415.9 & 423.4 & 416.51 & -0.62 & 7.52 \\
\hline 233. & 64 & 65 & 61.37 & 2.63 & 1.00 & 233. & 415.9 & 417.4 & 411.17 & 4.72 & 1.55 \\
\hline 234. & 54 & 57 & 54.29 & -0.29 & 3.00 & 234. & 398.9 & 404.3 & 399.42 & -0.53 & 5.41 \\
\hline 235. & 59 & 63 & 59.60 & -0.60 & 4.00 & 235. & 407.8 & 414.3 & 408.37 & -0.62 & 6.56 \\
\hline 236. & 64 & 69 & 64.91 & -0.91 & 5.00 & 236. & 415.9 & 423.4 & 416.51 & -0.62 & 7.52 \\
\hline 237. & 62 & 65 & 61.37 & 0.63 & 3.00 & 237. & 412.7 & 417.4 & 411.17 & 1.55 & 4.73 \\
\hline 238. & 37 & 32 & 32.17 & 4.83 & -5.00 & 238. & 361.1 & 346.6 & 347.80 & 13.29 & -14.52 \\
\hline 239. & 36 & 37 & 36.59 & -0.59 & 1.00 & 239. & 358.4 & 361.1 & 360.78 & -2.43 & 2.74 \\
\hline 240. & 42 & 45 & 43.67 & -1.67 & 3.00 & 240. & 373.8 & 380.7 & 378.29 & -4.52 & 6.90 \\
\hline
\end{tabular}


Development, Item Analysis and Standardization of Teachers Cognitive Ability Test

\begin{tabular}{|c|c|c|c|c|c|c|c|c|c|c|c|}
\hline 241. & 49 & 51 & 48.98 & 0.02 & 2.00 & 241. & 389.2 & 393.2 & 389.48 & -0.30 & 4.00 \\
\hline 242. & 52 & 58 & 55.18 & -3.18 & 6.00 & 242. & 395.1 & 406.0 & 400.98 & -5.85 & 10.92 \\
\hline 243. & 35 & 33 & 33.05 & 1.95 & -2.00 & 243. & 355.5 & 349.7 & 350.55 & 4.98 & -5.88 \\
\hline 244. & 52 & 58 & 55.18 & -3.18 & 6.00 & 244. & 395.1 & 406.0 & 400.98 & -5.85 & 10.92 \\
\hline 245. & 48 & 58 & 55.18 & -7.18 & 10.00 & 245. & 387.1 & 406.0 & 400.98 & $\begin{array}{l}-13.86 \\
\end{array}$ & 18.92 \\
\hline 246. & 59 & 63 & 59.60 & -0.60 & 4.00 & 246. & 407.8 & 414.3 & 408.37 & -0.62 & 6.56 \\
\hline 247. & 46 & 45 & 43.67 & 2.33 & -1.00 & 247. & 382.9 & 380.7 & 378.29 & 4.58 & -2.20 \\
\hline 248. & 43 & 45 & 43.67 & -0.67 & 2.00 & 248. & 376.1 & 380.7 & 378.29 & -2.17 & 4.55 \\
\hline 249. & 47 & 48 & 46.33 & 0.67 & 1.00 & 249. & 385.0 & 387.1 & 384.06 & 0.96 & 2.11 \\
\hline 250. & 42 & 48 & 46.33 & -4.33 & 6.00 & 250. & 373.8 & 387.1 & 384.06 & -10.29 & 13.35 \\
\hline 251. & 48 & 53 & 50.75 & -2.75 & 5.00 & 251. & 387.1 & 397.0 & 392.92 & -5.80 & 9.91 \\
\hline 252. & 49 & 53 & 50.75 & -1.75 & 4.00 & 252. & 389.2 & 397.0 & 392.92 & -3.74 & 7.85 \\
\hline 253. & 50 & 53 & 50.75 & -0.75 & 3.00 & 253. & 391.2 & 397.0 & 392.92 & -1.72 & 5.83 \\
\hline 254. & 51 & 55 & 52.52 & -1.52 & 4.00 & 254. & 393.2 & 400.7 & 396.23 & -3.05 & 7.55 \\
\hline 255. & 52 & 55 & 52.52 & -0.52 & 3.00 & 255. & 395.1 & 400.7 & 396.23 & -1.11 & 5.61 \\
\hline 256. & 54 & 58 & 55.18 & -1.18 & 4.00 & 256. & 398.9 & 406.0 & 400.98 & -2.08 & 7.15 \\
\hline 257. & 53 & 58 & 55.18 & -2.18 & 5.00 & 257. & 397.0 & 406.0 & 400.98 & -3.95 & 9.02 \\
\hline 258. & 71 & 65 & 61.37 & 9.63 & -6.00 & 258. & 426.3 & 417.4 & 411.17 & 15.10 & -8.83 \\
\hline 259. & 66 & 64 & 60.49 & 5.51 & -2.00 & 259. & 419.0 & 415.9 & 409.78 & 9.18 & -3.08 \\
\hline 260. & 37 & 37 & 36.59 & 0.41 & 0.00 & 260. & 361.1 & 361.1 & 360.78 & $\begin{array}{l}0.31 \\
\end{array}$ & 0.00 \\
\hline 261. & 53 & 55 & 52.52 & 0.48 & 2.00 & 261. & 397.0 & 400.7 & 396.23 & 0.80 & 3.70 \\
\hline 262. & 62 & 67 & 63.14 & -1.14 & 5.00 & 262. & 412.7 & 420.5 & 413.88 & -1.16 & 7.76 \\
\hline 263. & 67 & 71 & 66.68 & 0.32 & 4.00 & 263. & 420.5 & 426.3 & 419.06 & 1.41 & 5.80 \\
\hline 264. & 69 & 62 & 58.72 & 10.28 & -7.00 & 264. & 423.4 & 412.7 & 406.94 & 16.47 & -10.70 \\
\hline 265. & 62 & 62 & 58.72 & 3.28 & 0.00 & 265. & 412.7 & 412.7 & 406.94 & 5.77 & 0.00 \\
\hline 266. & 43 & 45 & 43.67 & -0.67 & 2.00 & 266. & 376.1 & 380.7 & 378.29 & -2.17 & 4.55 \\
\hline 267. & 47 & 48 & 46.33 & $\begin{array}{l}0.67 \\
\end{array}$ & 1.00 & 267. & 385.0 & 387.1 & 384.06 & 0.96 & 2.11 \\
\hline 268. & 42 & 48 & 46.33 & -4.33 & 6.00 & 268. & $\begin{array}{l}373.8 \\
\end{array}$ & 387.1 & 384.06 & -10.29 & 13.35 \\
\hline 269. & 48 & 53 & 50.75 & -2.75 & 5.00 & 269. & 387.1 & 397.0 & 392.92 & -5.80 & 9.91 \\
\hline 270. & 49 & 53 & 50.75 & -1.75 & 4.00 & 270. & 389.2 & 397.0 & 392.92 & -3.74 & 7.85 \\
\hline 271. & 50 & 53 & 50.75 & -0.75 & 3.00 & 271. & 391.2 & 397.0 & 392.92 & -1.72 & 5.83 \\
\hline 272. & 51 & 55 & 52.52 & -1.52 & 4.00 & 272. & 393.2 & 400.7 & 396.23 & -3.05 & 7.55 \\
\hline 273. & 52 & 55 & 52.52 & -0.52 & 3.00 & 273. & 395.1 & 400.7 & 396.23 & -1.11 & 5.61 \\
\hline 274. & 54 & 58 & 55.18 & -1.18 & 4.00 & 274. & 398.9 & 406.0 & 400.98 & -2.08 & 7.15 \\
\hline 275. & 53 & 58 & 55.18 & -2.18 & 5.00 & 275. & 397.0 & 406.0 & 400.98 & -3.95 & 9.02 \\
\hline 276. & 53 & 58 & 55.18 & -2.18 & 5.00 & 276. & 397.0 & 406.0 & 400.98 & -3.95 & 9.02 \\
\hline 277. & 56 & 58 & 55.18 & 0.82 & 2.00 & 277. & 402.5 & 406.0 & 400.98 & 1.56 & 3.51 \\
\hline 278. & 58 & 59 & 56.06 & 1.94 & 1.00 & 278. & 406.0 & 407.8 & 402.51 & 3.54 & 1.71 \\
\hline 279. & 54 & 60 & 56.95 & -2.95 & 6.00 & 279. & 398.9 & 409.4 & 404.01 & -5.11 & 10.54 \\
\hline 280. & 57 & 60 & 56.95 & 0.05 & 3.00 & 280. & 404.3 & 409.4 & 404.01 & 0.29 & 5.13 \\
\hline 281. & 59 & 62 & 58.72 & 0.28 & 3.00 & 281. & 407.8 & 412.7 & 406.94 & 0.81 & 4.96 \\
\hline 282. & 60 & 63 & 59.60 & 0.40 & 3.00 & 282. & 409.4 & 414.3 & 408.37 & 1.06 & 4.88 \\
\hline 283. & 59 & 67 & 63.14 & -4.14 & 8.00 & 283. & 407.8 & 420.5 & 413.88 & -6.12 & 12.72 \\
\hline 284. & 58 & 68 & 64.03 & -6.03 & 10.00 & 284. & 406.0 & 422.0 & 415.20 & -9.16 & 15.91 \\
\hline 285. & 66 & 69 & 64.91 & 1.09 & 3.00 & 285. & 419.0 & 423.4 & 416.51 & 2.46 & 4.45 \\
\hline 286. & 55 & 59 & 56.06 & -1.06 & 4.00 & 286. & 400.7 & 407.8 & 402.51 & -1.77 & 7.02 \\
\hline 287. & 62 & 66 & 62.26 & -0.26 & 4.00 & 287. & 412.7 & 419.0 & 412.53 & 0.18 & 6.25 \\
\hline 288. & 60 & 69 & 64.91 & -4.91 & 9.00 & 288. & 409.4 & 423.4 & 416.51 & -7.07 & 13.98 \\
\hline 289. & 65 & 69 & 64.91 & 0.09 & 4.00 & 289. & 417.4 & 423.4 & 416.51 & 0.93 & 5.97 \\
\hline
\end{tabular}


Development, Item Analysis and Standardization of Teachers Cognitive Ability Test

\begin{tabular}{|c|c|c|c|c|c|c|c|c|c|c|c|}
\hline 290. & 64 & 69 & 64.91 & -0.91 & 5.00 & 290. & 415.9 & 423.4 & 416.51 & -0.62 & 7.52 \\
\hline 291. & 62 & 66 & 62.26 & -0.26 & 4.00 & 291. & 412.7 & 419.0 & 412.53 & 0.18 & 6.25 \\
\hline 292. & 54 & 57 & 54.29 & -0.29 & 3.00 & 292. & 398.9 & 404.3 & 399.42 & -0.53 & 5.41 \\
\hline 293. & 59 & 63 & 59.60 & -0.60 & 4.00 & 293. & 407.8 & 414.3 & 408.37 & $\begin{array}{l}-0.62 \\
\end{array}$ & 6.56 \\
\hline 294. & 64 & 69 & 64.91 & -0.91 & 5.00 & 294. & 415.9 & 423.4 & 416.51 & -0.62 & 7.52 \\
\hline 295. & 62 & 65 & 61.37 & 0.63 & 3.00 & 295. & 412.7 & 417.4 & 411.17 & 1.55 & 4.73 \\
\hline 296. & 37 & 32 & 32.17 & 4.83 & -5.00 & 296. & 361.1 & 346.6 & 347.80 & 13.29 & -14.52 \\
\hline 297. & 36 & 37 & 36.59 & -0.59 & 1.00 & 297. & 358.4 & 361.1 & 360.78 & -2.43 & 2.74 \\
\hline 298. & 42 & 45 & 43.67 & -1.67 & 3.00 & 298. & 373.8 & 380.7 & 378.29 & -4.52 & 6.90 \\
\hline 299. & 49 & 51 & 48.98 & 0.02 & 2.00 & 299. & 389.2 & 393.2 & 389.48 & -0.30 & 4.00 \\
\hline 300. & 55 & 59 & 56.06 & -1.06 & 4.00 & 300. & 400.7 & 407.8 & 402.51 & -1.77 & 7.02 \\
\hline 301. & 57 & 60 & 56.95 & 0.05 & 3.00 & 301. & 404.3 & 409.4 & 404.01 & 0.29 & 5.13 \\
\hline 302. & 65 & 64 & 60.49 & 4.51 & -1.00 & 302. & 417.4 & 415.9 & 409.78 & 7.66 & -1.55 \\
\hline 303. & 60 & 68 & 64.03 & -4.03 & 8.00 & 303. & 409.4 & 422.0 & 415.20 & -5.77 & 12.52 \\
\hline 304. & 68 & 66 & 62.26 & 5.74 & -2.00 & 304. & 422.0 & 419.0 & 412.53 & 9.42 & -2.99 \\
\hline 305. & 66 & 66 & 62.26 & 3.74 & 0.00 & 305. & 419.0 & 419.0 & 412.53 & 6.43 & 0.00 \\
\hline 306. & 66 & 68 & 64.03 & 1.97 & 2.00 & 306. & 419.0 & 422.0 & 415.20 & 3.76 & 2.99 \\
\hline 307. & 66 & 69 & 64.91 & 1.09 & 3.00 & 307. & 419.0 & 423.4 & 416.51 & 2.46 & 4.45 \\
\hline 308. & 66 & 67 & 63.14 & 2.86 & 1.00 & 308. & 419.0 & 420.5 & 413.88 & 5.09 & 1.50 \\
\hline 309. & 67 & 63 & 59.60 & 7.40 & -4.00 & 309. & 420.5 & 414.3 & 408.37 & 12.10 & -6.16 \\
\hline 310. & 51 & 59 & 56.06 & -5.06 & 8.00 & 310. & 393.2 & 407.8 & 402.51 & -9.32 & 14.57 \\
\hline 311. & 63 & 69 & 64.91 & -1.91 & 6.00 & 311. & 414.3 & 423.4 & 416.51 & -2.19 & 9.10 \\
\hline 312. & 62 & 67 & 63.14 & -1.14 & 5.00 & 312. & 412.7 & 420.5 & 413.88 & -1.16 & 7.76 \\
\hline 313. & 48 & 50 & 48.10 & -0.10 & 2.00 & 313. & 387.1 & 391.2 & 387.71 & $\begin{array}{l}-0.59 \\
\end{array}$ & 4.08 \\
\hline 314. & 50 & 50 & 48.10 & 1.90 & 0.00 & 314. & 391.2 & 391.2 & 387.71 & 3.49 & 0.00 \\
\hline 315. & 55 & 59 & 56.06 & -1.06 & 4.00 & 315. & 400.7 & 407.8 & 402.51 & -1.77 & 7.02 \\
\hline 316. & 50 & 55 & 52.52 & -2.52 & 5.00 & 316. & 391.2 & 400.7 & 396.23 & -5.03 & 9.53 \\
\hline 317. & 54 & 58 & 55.18 & -1.18 & 4.00 & 317. & 398.9 & 406.0 & 400.98 & -2.08 & 7.15 \\
\hline 318. & 66 & 72 & 67.57 & -1.57 & 6.00 & 318. & 419.0 & 427.7 & 420.31 & -1.35 & 8.70 \\
\hline 319. & 36 & 35 & 34.82 & 1.18 & -1.00 & 319. & 358.4 & 355.5 & 355.82 & 2.54 & -2.82 \\
\hline 320. & 48 & 50 & 48.10 & -0.10 & 2.00 & 320. & 387.1 & 391.2 & 387.71 & -0.59 & 4.08 \\
\hline 321. & 58 & 62 & 58.72 & -0.72 & 4.00 & 321. & 406.0 & 412.7 & 406.94 & -0.90 & 6.67 \\
\hline 322. & 52 & 54 & 51.64 & 0.36 & 2.00 & 322. & 395.1 & 398.9 & 394.59 & 0.53 & 3.77 \\
\hline 323. & 46 & 51 & 48.98 & -2.98 & 5.00 & 323. & 382.9 & 393.2 & 389.48 & -6.61 & 10.32 \\
\hline 324. & 49 & 50 & 48.10 & 0.90 & 1.00 & 324. & 389.2 & 391.2 & 387.71 & 1.47 & 2.02 \\
\hline 325. & 65 & 64 & 60.49 & 4.51 & -1.00 & 325. & 417.4 & 415.9 & 409.78 & 7.66 & -1.55 \\
\hline 326. & 39 & 41 & 40.13 & -1.13 & 2.00 & 326. & 366.4 & 371.4 & 369.96 & -3.61 & 5.00 \\
\hline 327. & 52 & 57 & 54.29 & -2.29 & 5.00 & 327. & 395.1 & 404.3 & 399.42 & -4.30 & 9.18 \\
\hline 328. & 71 & 62 & 58.72 & 12.28 & -9.00 & 328. & 426.3 & 412.7 & 406.94 & 19.33 & -13.55 \\
\hline 329. & 70 & 64 & 60.49 & 9.51 & -6.00 & 329. & 424.8 & 415.9 & 409.78 & 15.07 & -8.96 \\
\hline 330. & 37 & 41 & 40.13 & -3.13 & 4.00 & 330. & 361.1 & 371.4 & 369.96 & -8.87 & 10.27 \\
\hline 331. & 46 & 45 & 43.67 & 2.33 & -1.00 & 331. & 382.9 & 380.7 & 378.29 & 4.58 & -2.20 \\
\hline 332. & 43 & 45 & 43.67 & -0.67 & 2.00 & 332. & 376.1 & 380.7 & 378.29 & -2.17 & 4.55 \\
\hline 333. & 47 & 48 & 46.33 & 0.67 & 1.00 & 333. & 385.0 & 387.1 & 384.06 & 0.96 & 2.11 \\
\hline 334. & 42 & 48 & 46.33 & -4.33 & 6.00 & 334. & 373.8 & 387.1 & 384.06 & -10.29 & 13.35 \\
\hline 335. & 48 & 53 & 50.75 & -2.75 & 5.00 & 335. & 387.1 & 397.0 & 392.92 & -5.80 & 9.91 \\
\hline 336. & 49 & 53 & 50.75 & -1.75 & 4.00 & 336. & 389.2 & 397.0 & 392.92 & -3.74 & 7.85 \\
\hline 337. & 50 & 53 & 50.75 & -0.75 & 3.00 & 337. & 391.2 & 397.0 & 392.92 & -1.72 & 5.83 \\
\hline 338. & 51 & 55 & 52.52 & -1.52 & 4.00 & 338. & 393.2 & 400.7 & 396.23 & -3.05 & 7.55 \\
\hline
\end{tabular}


Development, Item Analysis and Standardization of Teachers Cognitive Ability Test

\begin{tabular}{|c|c|c|c|c|c|c|c|c|c|c|c|}
\hline 339. & 52 & 55 & 52.52 & -0.52 & 3.00 & 339. & 395.1 & 400.7 & 396.23 & -1.11 & 5.61 \\
\hline 340. & 54 & 58 & 55.18 & -1.18 & 4.00 & 340. & 398.9 & 406.0 & 400.98 & -2.08 & 7.15 \\
\hline 341. & 53 & 58 & 55.18 & -2.18 & 5.00 & 341. & 397.0 & 406.0 & 400.98 & -3.95 & 9.02 \\
\hline 342. & 53 & 58 & 55.18 & -2.18 & 5.00 & 342. & 397.0 & 406.0 & 400.98 & -3.95 & 9.02 \\
\hline 343. & 56 & 58 & 55.18 & 0.82 & 2.00 & 343. & 402.5 & 406.0 & 400.98 & 1.56 & 3.51 \\
\hline 344. & 58 & 59 & 56.06 & 1.94 & 1.00 & 344. & 406.0 & 407.8 & 402.51 & 3.54 & 1.71 \\
\hline 345. & 54 & 60 & 56.95 & -2.95 & 6.00 & 345. & 398.9 & 409.4 & 404.01 & -5.11 & 10.54 \\
\hline 346. & 57 & 60 & 56.95 & 0.05 & 3.00 & 346. & 404.3 & 409.4 & 404.01 & 0.29 & 5.13 \\
\hline 347. & 59 & 62 & 58.72 & 0.28 & 3.00 & 347. & 407.8 & 412.7 & 406.94 & 0.81 & 4.96 \\
\hline 348. & 60 & 63 & 59.60 & 0.40 & 3.00 & 348. & 409.4 & 414.3 & 408.37 & 1.06 & 4.88 \\
\hline 349. & 59 & 67 & 63.14 & -4.14 & 8.00 & 349. & 407.8 & 420.5 & 413.88 & -6.12 & 12.72 \\
\hline 350. & 58 & 68 & 64.03 & -6.03 & 10.00 & 350. & 406.0 & 422.0 & 415.20 & -9.16 & 15.91 \\
\hline 351. & 66 & 69 & 64.91 & 1.09 & 3.00 & 351. & 419.0 & 423.4 & 416.51 & 2.46 & 4.45 \\
\hline 352. & 55 & 59 & 56.06 & -1.06 & 4.00 & 352. & 400.7 & 407.8 & 402.51 & -1.77 & 7.02 \\
\hline 353. & 62 & 66 & 62.26 & -0.26 & 4.00 & 353. & 412.7 & 419.0 & 412.53 & 0.18 & 6.25 \\
\hline 354. & 60 & 69 & 64.91 & -4.91 & 9.00 & 354. & 409.4 & 423.4 & 416.51 & -7.07 & 13.98 \\
\hline 355. & 65 & 69 & 64.91 & 0.09 & 4.00 & 355. & 417.4 & 423.4 & 416.51 & 0.93 & 5.97 \\
\hline 356. & 64 & 69 & 64.91 & -0.91 & 5.00 & 356. & 415.9 & 423.4 & 416.51 & -0.62 & 7.52 \\
\hline 357. & 61 & 67 & 63.14 & -2.14 & 6.00 & 357. & 411.1 & 420.5 & 413.88 & -2.79 & 9.38 \\
\hline 358. & 54 & 57 & 54.29 & -0.29 & 3.00 & 358. & 398.9 & 404.3 & 399.42 & -0.53 & 5.41 \\
\hline 359. & 59 & 63 & 59.60 & -0.60 & 4.00 & 359. & 407.8 & $\begin{array}{l}414.3 \\
\end{array}$ & 408.37 & -0.62 & 6.56 \\
\hline 360. & 64 & 69 & 64.91 & -0.91 & 5.00 & 360. & 415.9 & 423.4 & 416.51 & -0.62 & 7.52 \\
\hline 361. & 62 & 65 & 61.37 & 0.63 & 3.00 & 361. & 412.7 & 417.4 & 411.17 & 1.55 & 4.73 \\
\hline 362. & 37 & 32 & 32.17 & 4.83 & -5.00 & 362. & 361.1 & 346.6 & 347.80 & 13.29 & -14.52 \\
\hline 363. & 36 & 37 & 36.59 & -0.59 & 1.00 & 363. & 358.4 & 361.1 & 360.78 & -2.43 & 2.74 \\
\hline 364. & 42 & 45 & 43.67 & -1.67 & 3.00 & 364. & 373.8 & 380.7 & 378.29 & -4.52 & 6.90 \\
\hline 365. & 49 & 51 & 48.98 & 0.02 & 2.00 & 365. & 389.2 & 393.2 & $\begin{array}{l}389.48 \\
\end{array}$ & -0.30 & 4.00 \\
\hline 366. & 52 & 58 & 55.18 & -3.18 & 6.00 & 366. & 395.1 & 406.0 & 400.98 & -5.85 & 10.92 \\
\hline 367. & 48 & 58 & 55.18 & -7.18 & 10.00 & 367. & 387.1 & 406.0 & 400.98 & -13.86 & 18.92 \\
\hline 368. & 59 & 63 & 59.60 & -0.60 & 4.00 & 368. & 407.8 & 414.3 & 408.37 & -0.62 & 6.56 \\
\hline 369. & 47 & 48 & 46.33 & 0.67 & 1.00 & 369. & 385.0 & 387.1 & 384.06 & 0.96 & 2.11 \\
\hline 370. & 41 & 44 & 42.79 & -1.79 & 3.00 & 370. & 371.4 & 378.4 & 376.28 & -4.92 & 7.06 \\
\hline 371. & 50 & 55 & 52.52 & -2.52 & 5.00 & 371. & 391.2 & 400.7 & 396.23 & -5.03 & 9.53 \\
\hline 372. & 60 & 63 & 59.60 & 0.40 & 3.00 & 372. & 409.4 & 414.3 & 408.37 & 1.06 & 4.88 \\
\hline 373. & 62 & 67 & 63.14 & -1.14 & 5.00 & 373. & 412.7 & 420.5 & 413.88 & -1.16 & 7.76 \\
\hline 374. & 48 & 50 & 48.10 & -0.10 & 2.00 & 374. & 387.1 & 391.2 & 387.71 & -0.59 & 4.08 \\
\hline 375. & 50 & 50 & 48.10 & 1.90 & 0.00 & 375. & 391.2 & 391.2 & 387.71 & 3.49 & 0.00 \\
\hline 376. & 55 & 59 & 56.06 & -1.06 & 4.00 & 376. & 400.7 & 407.8 & 402.51 & -1.77 & 7.02 \\
\hline 377. & 50 & 55 & 52.52 & -2.52 & 5.00 & 377. & 391.2 & 400.7 & 396.23 & -5.03 & 9.53 \\
\hline 378. & 54 & 58 & 55.18 & -1.18 & 4.00 & 378. & 398.9 & 406.0 & 400.98 & -2.08 & 7.15 \\
\hline 379. & 66 & 72 & 67.57 & -1.57 & 6.00 & 379. & 419.0 & 427.7 & 420.31 & -1.35 & 8.70 \\
\hline 380. & 36 & 35 & 34.82 & 1.18 & -1.00 & 380. & 358.4 & 355.5 & 355.82 & 2.54 & -2.82 \\
\hline 381. & 48 & 50 & 48.10 & -0.10 & 2.00 & 381. & 387.1 & 391.2 & 387.71 & -0.59 & 4.08 \\
\hline 382. & 58 & 62 & 58.72 & -0.72 & 4.00 & 382. & 406.0 & 412.7 & 406.94 & -0.90 & 6.67 \\
\hline 383. & 52 & 54 & 51.64 & 0.36 & 2.00 & 383. & 395.1 & 398.9 & 394.59 & 0.53 & -3.77 \\
\hline Mean & 54.9 & 57.6 & & & 2.78 & $\begin{array}{r}\text { Back-trans } \\
\text { mean }\end{array}$ & 54.1 & 56.8 & & & 4.9 \\
\hline SD & 8.9 & 9.4 & & & 3.41 & $\begin{array}{r}\text { SD as a CV } \\
(\%)\end{array}$ & 18.9 & 20.0 & & & 6.2 \\
\hline $\mathrm{N}$ & 383 & 383 & & & & $\mathrm{~N}$ & 383 & 383 & & & \\
\hline$\Sigma \mathrm{X}^{2}$ & & 33606 & & & & $\Sigma \mathrm{X}^{2}$ & & 127205 & & & \\
\hline
\end{tabular}




\begin{tabular}{|c|c|c|c|c|c|c|c|c|c|c|c|}
\hline $\begin{array}{r}\text { Calibratio } \\
\text { n equation: } \\
\mathbf{Y}= \\
\text { intercept + } \\
\text { slope*X } \\
\end{array}$ & Estimate & $\begin{array}{c}\text { Lower } \\
\text { CL }\end{array}$ & $\begin{array}{c}\text { Upper } \\
\text { CL }\end{array}$ & $\pm \mathrm{CL}$ & $\begin{array}{c}\text { approx } \\
\times \\
\times / \div C L\end{array}$ & $\begin{array}{r}\text { Calibratio } \\
\text { n equation: } \\
\mathbf{Y}=\mathbf{a X}^{\wedge} \mathbf{b}\end{array}$ & Estimate & $\begin{array}{l}\text { Lower } \\
\text { CL }\end{array}$ & $\begin{array}{l}\text { Upper } \\
\text { CL }\end{array}$ & $\begin{array}{l}\times / \div \\
C L\end{array}$ & $\stackrel{ \pm}{\mathrm{CL}}$ \\
\hline intercept & 3.850 & 1.823 & 5.878 & 2.027 & & $\mathrm{a}$ & 1.461 & 1.286 & 1.659 & 1.14 & - \\
\hline slope & 0.885 & 0.850 & 0.920 & 0.035 & & $\mathrm{~b}$ & 0.894 & 0.863 & 0.926 & - & 0.031 \\
\hline $\begin{array}{l}\text { Enter an X } \\
\text { value here: }\end{array}$ & 62.00 & & & & & $\begin{array}{l}\text { Enter an } \mathrm{X} \\
\text { value here: }\end{array}$ & 380.00 & & & & \\
\hline $\begin{array}{r}\text { Predicted } \\
\text { (estimated) } \\
\mathrm{Y} \text { at X }\end{array}$ & 58.72 & 52.34 & 65.09 & 6.37 & & $\begin{array}{r}\text { Predicted } \\
\text { (estimated) } \\
\mathrm{Y} \text { at X }\end{array}$ & 296.06 & $\begin{array}{c}260.6 \\
4\end{array}$ & 336.29 & 1.14 & \\
\hline $\begin{array}{r}\text { Bias at } X \\
\text { value } \\
\end{array}$ & & & & & & $\begin{array}{r}\text { Bias at } X \\
\text { value } \\
\end{array}$ & & & & & \\
\hline in raw units & 3.28 & 2.92 & 3.64 & 0.36 & & as a $\%$ & 28.4 & 20.9 & 36.3 & - & 7.7 \\
\hline $\begin{array}{r}\text { standardize } \\
\mathrm{d}\end{array}$ & 0.37 & 0.33 & 0.41 & 0.04 & & as a factor & 1.284 & 1.209 & 1.363 & 1.062 & - \\
\hline & & & & & & $\begin{array}{r}\text { standardize } \\
\mathrm{d}\end{array}$ & 1.44 & 1.10 & 1.79 & - & 0.35 \\
\hline $\begin{array}{r}\begin{array}{r}\text { Overall } \\
\text { bias }\end{array} \\
\end{array}$ & & & & & & $\begin{array}{r}\begin{array}{r}\text { Overall } \\
\text { bias }\end{array} \\
\end{array}$ & & & & & \\
\hline $\begin{array}{r}\text { Mean bias } \\
\text { in raw units }\end{array}$ & 2.78 & 2.44 & 3.12 & 0.34 & & $\begin{array}{r}\text { Mean bias } \\
\text { as a \% } \\
\end{array}$ & 5.1 & 4.3 & 5.6 & - & 0.6 \\
\hline $\begin{array}{r}\text { Mean bias } \\
\text { standardize } \\
\mathrm{d}\end{array}$ & 0.31 & 0.27 & 0.35 & 0.04 & & $\begin{array}{l}\text { Mean bias } \\
\text { as a factor }\end{array}$ & 1.051 & 1.043 & 1.056 & 1.006 & - \\
\hline $\begin{array}{l}\text { SD of bias } \\
\text { in raw units }\end{array}$ & 3.41 & 3.18 & 3.67 & & 1.07 & $\begin{array}{r}\text { Mean bias } \\
\text { standardize } \\
\mathrm{d}\end{array}$ & 0.28 & 0.24 & 0.31 & - & 0.04 \\
\hline $\begin{array}{r}\text { SD of bias } \\
\text { standardize } \\
\mathrm{d} \\
\end{array}$ & 0.38 & 0.36 & 0.41 & & 1.07 & $\begin{array}{r}\text { SD of bias } \\
\text { as a \% } \\
\end{array}$ & 6.4 & 6.0 & 6.9 & 1.08 & \\
\hline & & & & & & $\begin{array}{l}\text { SD of bias } \\
\text { as a factor }\end{array}$ & 1.064 & 1.060 & 1.069 & 1.004 & \\
\hline & & & & & & $\begin{array}{r}\text { SD of bias } \\
\text { standardize } \\
\mathrm{d}\end{array}$ & 0.36 & 0.34 & 0.39 & 1.07 & \\
\hline $\begin{array}{r}\text { Typical } \\
\text { error of } \\
\text { estimate }\end{array}$ & $\begin{array}{c}\text { Estimat } \\
\mathrm{e}\end{array}$ & $\begin{array}{c}\text { Lowe } \\
\mathrm{r} \\
\mathrm{CL} \\
\end{array}$ & $\begin{array}{c}\text { Upper } \\
\text { CL }\end{array}$ & & & $\begin{array}{r}\text { Typical } \\
\text { error of } \\
\text { estimate }\end{array}$ & Estimate & $\begin{array}{c}\text { Lower } \\
\text { CL }\end{array}$ & $\begin{array}{c}\text { Upper } \\
\text { CL }\end{array}$ & $\begin{array}{c}\text { appro } \\
\mathrm{X} . \\
\times / \div \mathrm{CL} \\
\end{array}$ & \\
\hline in raw units & 3.24 & 3.02 & 3.48 & & 1.07 & $\begin{array}{r}\text { as a CV } \\
(\%)\end{array}$ & 5.9 & 5.5 & 6.3 & 1.08 & \\
\hline $\begin{array}{r}\text { standardize } \\
\mathrm{d} \\
\end{array}$ & 0.36 & 0.34 & 0.39 & & 1.07 & $\begin{array}{r}\text { as a } \times / \div \\
\text { factor }\end{array}$ & 1.059 & 1.055 & 1.063 & 1.004 & \\
\hline \multirow[t]{3}{*}{$\begin{array}{r}\text { PRRES } \\
\text { error } \\
\end{array}$} & 10.47 & & & & & $\begin{array}{r}\text { standardize } \\
\mathrm{d} \\
\end{array}$ & 0.33 & 0.31 & 0.36 & 1.07 & \\
\hline & & & & & & $\begin{array}{r}\text { PRRES } \\
\text { error (\%) }\end{array}$ & 5.9 & & & & \\
\hline & Estimate & $\begin{array}{c}\text { Lower } \\
\text { CL }\end{array}$ & $\begin{array}{c}\text { Upper } \\
\text { CL }\end{array}$ & $\begin{array}{c}\text { approx } \\
. \pm \mathrm{CL}\end{array}$ & & & Estimate & $\begin{array}{l}\text { Lower } \\
\text { CL }\end{array}$ & $\begin{array}{c}\text { Upper } \\
\text { CL }\end{array}$ & & $\begin{array}{c}\text { appro } \\
\text { X. } \\
\pm \mathrm{CL} \\
\end{array}$ \\
\hline $\begin{array}{r}\text { Pearson } \\
\text { correlation }\end{array}$ & 0.93 & 0.92 & 0.94 & 0.01 & & $\begin{array}{r}\begin{array}{r}\text { Pearson } \\
\text { correlation }\end{array} \\
\end{array}$ & 0.94 & 0.93 & 0.95 & & 0.01 \\
\hline $\begin{array}{r}\text { Bland- } \\
\text { Altman } \\
\end{array}$ & Estimate & & & & & $\begin{array}{r}\text { Bland- } \\
\text { Altman } \\
\end{array}$ & Estimate & & & & \\
\hline $\begin{array}{r}95 \% \text { limits } \\
\text { of } \\
\text { agreement }\end{array}$ & 0.01 & & & & & $\begin{array}{r}95 \% \text { limits } \\
\text { of } \\
\text { agreement } \\
(\%)\end{array}$ & 12.2 & & & & \\
\hline $\begin{array}{l}\text { "Total" or } \\
\text { "pure" error }\end{array}$ & 0.01 & & & & & $\begin{array}{r}\text { "Total" or } \\
\text { "pure" error } \\
(\%)\end{array}$ & 6.2 & & & & \\
\hline
\end{tabular}


Development, Item Analysis and Standardization of Teachers Cognitive Ability Test

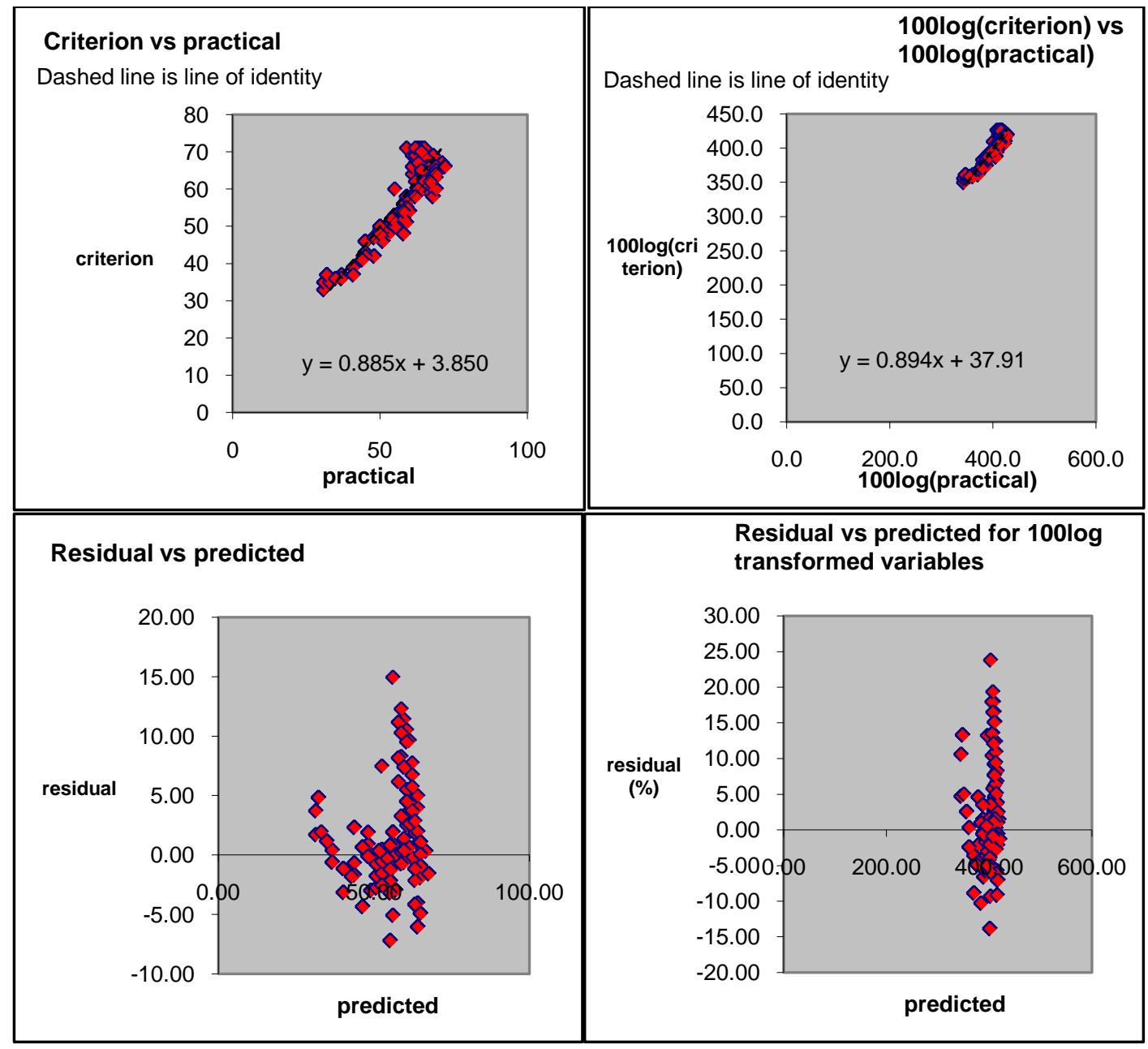

Fig.3 Validity via Linear Regression Method

\section{CONCLUSION}

In a nutshell, the study came out with significant results as the correlation coefficient was found to be significantly high witnessing the high reliability and validity of the test. It was notified that there are many factors along with IQ that have a great impact on the individual. These factors like Focus, Decision Making Ability, Creativity, Passion, Judgment, Estimation Level, and Nature of Work \& Professional Choice must be taken into consideration being more psychological nature than statistical. The study aided in the development \& standardization of Development, item analysis and standardization of the Teachers Cognitive Ability Test. The objective of the research was achieved as a product intended to provide an insight into those scientific methodologies that can help us measure and reorder human intelligence to enhance cognitive factors among teachers by filling the gaps, to produce successful and efficient teachers. The test would help in the identification, measurement and analysis of core cognitive ability factors that determine success in teaching. The reliability was estimated through Test-Retest Method (Table 9 and Fig 2). The validity was estimated via linear regression method (Table 10,11 and Fig 3). The study came out with significant outputs as the correlation coefficient was 
found to be significantly high witnessing the high validity and reliability of the test.

\section{ACKNOWLEDGEMENT}

Authors express indebtedness to the Almighty, who is the apostle of strength. Authors are inevitably grateful to the Institutions, all the subjects and all those directly as well as indirectly involved in the auspicious research work. Genuine thanks are expressed to all the authors/researches whose work is referred for making the present study a real success.

\section{BIBLIOGRAPHY}

Atkinson, Hilgard. Introduction Psychology. Harcourt Brace Publishers, America, 1995, 399.

Ball, R. S. 1938. "The Predictability of Occupational Level From Intelligence." Journal of Consulting Psychology 2:184-86.

Bar-Yosef, C., Weinblatt, N., \& Katz, N. (1999). Reliability and validity of the Cognitive Performance Test (CPT) in an elderly population in Israel. Physical and Occupational Therapy in Geriatrics, 17(1), 65-79.

Betsy Moore, Toddy Stanley, Critical \& Formative Thinking Assessment, Eye on Education, 2009,156. 6. Carroll B. Randy W. Kamphaus. Clinical Assessment of Child and Adolescent Intelligence, Springer Science \& Business Media. Psychology, 2005, 676. 7.

Bloom F, Nelson CA, Lazerson A. Brain, mind and behavior (3rd ed.). New York: Worth Publishers, 2001.

Bloom CM, Krathwhol DR. Taxonomy of educational objectives, Hardbook 1, Congnitive Domain. New York: David Mekay, 1956.

Borko, H., Putnam, R.T. (1995). "Expanding a Teacher's Knowledge Base: A Cognitive Psychological Perspective on Professional Development.” In T.R. Guskey and M. Huberman (Eds.) Professional Development in Education: New Paradigms and Practices, pp. 35-66.

Brown, C. (2000). Clinical interpretation of "A comparison of the Allen Cognitive Level Test and the Wisconsin Card Sorting Test in adults with schizophrenia". American Journal of Occupational Therapy, 54(2), 134-136.

Brophy, J.E., Good, T.L. (1986). “Teacher Behavior and Student Achievement.” In M.L. Wittrock (Ed.)Handbook of Research on Teaching, 3rd ed., pp. 328-375.

Bryk, Anthony. S., and Mary Erina. 1988. The High School as Community: Contextual Influences and Consequences for Students and Teachers. Madison, WI: National Center on Effective Secondary Schools, University of Wisconsin-Madison. Buddin, Richard, and GemaZamarro. 2009. Teacher qualifications and student achievement in urban elementary schools. Journal of Urban Economics 66(2): 103-115.

Clotfelter, Charles T., Helen F. Ladd, and Jacob L. Vigdor. 2006. Teacher-student matching and the assessment of teacher effectiveness. Journal of Human Resources 41(4): 778-820.

Coleman, James. S., Thomas B. Hoffer, and Sally Kilgore. 1982. Cognitive outcomes in public and private schools. Sociology of Education 55(2): 65-76. 
CT Dasen, P. (1994). Culture and cognitive development from a Piagetian perspective.

Fayetteville, AR: University of Arkansas. 29 Chetty, Raj., John N. Friedman, and Jonah E. Rockoff. 2014. Measuring the impacts of teachers II: Teacher value-added and student outcomes in adulthood. American Economic Review 104(9): 2633-2679.

Garet, M.S., et al. (2001). "What Makes Professional Development Effective? Results from a National Sample of Teachers.” American Educational Research Journal, Vol. 38, No. 4 (winter), pp. 915-945.

Goldhaber, Dan. 2008. Teachers Matter, But Effective Teacher Quality Policies are Elusive. In H. F. Ladd and E. B. Fiske (Eds.), Handbook of Research in Education Finance and Policy (pp. 146-165).

Goldhaber, Dan. 2007. Everyone's Doing it but What does Teacher Testing Tell us about Teacher Effectiveness. Journal of Human Resources 42(4): 765-794.

Hanushek, Eric A. 2011. The economic value of higher teacher quality. Economics of Education Review 30(3): 466-479.

Hanushek, Eric A., and Stevem G. Rivkin. 2006. Teacher Quality. In E. A. Hanushek and F. Welch (Eds.), Handbook of the Economics of Education (Vol. 2, pp. 1052-1078).

Kane, Thomas, Jonah E. Rockoff, and Douglas O. Staiger. 2008. What Does Certification Tell Us about Teacher Effectiveness. Economics of Education Review 27(6): 615-631.

Kyllonen, P.C., and J.P. Bertling. 2013. Innovative questionnaire assessment methods to increase cross-country comparability. In Handbook of international large-scale assessment data analysis: Background, technical issues, and methods of data analysis, edited by Leslie Rutkowski, Matthias von Davier, and David Rutkowski, 277-286.

Louis, K.S., Marks, H.M. (1998). "Does Professional Community Affect the Classroom? Teachers’ Work and Student Experiences in Restructuring Schools.” American Journal of Education, Vol. 106, pp. 532-575.

McCutchen, D., et al. (2002). "Beginning Literacy: Links among Teacher Knowledge, Teacher Practice, and Student Learning.” Journal of Learning Disabilities, Vol. 35, No. 1, 69-86.

Piaget, J. (1936). Origins of intelligence in the child. London: Routledge\&Kegan Paul.

Piaget, J., \& Cook, M. T. (1952). The origins of intelligence in children. New York, NY: International University Press.

Richardson, V. (1999). “Teacher Education and the Construction of Meaning.” In G. Griffin (Ed.) Teacher Education for a New Century: Emerging Perspectives, Promising Practices, and Future Possibilities (Yearbook of the National Society for the Study of Education), pp. 145-166.

Rosebery, A., Puttick, G.M. (1998). “Teacher Professional Development as Situated SenseMaking: A Case Study in Science Education.”Science Education, Vol. 82, pp. 649-677.

Velligan, D. I., Bow-Thomas, C. C., Mahurin, R., Miller, A., Dassori, A., \&Erdely, F. (1998).Concurrent and predictive validity of the Allen Cognitive Levels Assessment. Psychiatry Research,80(3), 287-298. 
Velligan, D. I., True, J. E., Lefton, R. S., Moore, T. C., \& Flores, C. V. (1995). Validity of the Allen Cognitive Levels Assessment: A tri-ethnic comparison. Psychiatry Research, 56(2), 101-109.

Voydetich, D., Jensen, M., Sigford, B., \&Mehr, J. (2002). Relationship between the Allen cognitive levels and the Rachos Los Amigos cognitive levels. Preimer Outlook, 3(4), 1419.

Wadsworth, B. J. (2004). Piaget's theory of cognitive and affective development: Foundations of constructivism. Longman Publishing.

Warren, John R., Jennifer T. Sheridan, and Robert M. Hauser. 2002. "Occupational Stratification Across the Life Course: Evidence From the Wisconsin Longitudinal Study." 67(3, June):432-55.

White, S., Meade, S., \&Hadar, L. (2007). Occupational therapy cognitive adaptation: An intervention in time management for persons with co-occurring conditions, OT Practice, June 25 (11), 9-14.

Wilson, S. D., Allen, C. D., McCormack, G., \& Burton, G. (1989). Cognitive disability and routine task behaviors in a community-based population with senile dementia. Occupational Therapy Practice, 1(1), 58-66.

Wilson, B. G. \& Myers, K. M. (1999). Situated Cognition in Theoretical and Practical Context. For inclusion in D. Jonassen\& S. Lands (Eds.), Theoretical Foundations of Learning Environments. Mahwah NJ: Erlbaum. 